\title{
Dynamic Birefringence Behavior of Semicrystalline Polymers. II. The Crystalline Relaxation Mechanisms of Low- and Medium-Density Polyethylenes*
}

\author{
Thein Kyu, ${ }^{* *}$ Naoshi Yasuda, Shoji Suehiro, Shunji Nomura, \\ and Hiromichi KAWAI*** \\ Department of Polymer Chemistry, Faculty of Engineering, \\ Kyoto University, Kyoto 606, Japan.
}

(Received May 17, 1976)

\begin{abstract}
The dynamic mechanical and birefringence behaviors of two kinds of polyethylenes, low- and medium-density polyethylenes having different thermal histories, were investigated over the ranges of frequency from 0.008 to $4.3 \mathrm{~Hz}$ and of temperature from 30 to $90^{\circ} \mathrm{C}$. Reducing the behavior at various temperatures to a given reference temperature of $50^{\circ} \mathrm{C}$, both behaviors showed rather broad frequency dispersions, corresponding to the $\alpha$ dispersion of the materials, with activation energies of the mechanical and optical relaxation processes of around $25 \mathrm{kcal} / \mathrm{mol}$ for every specimen tested. The behavior for a particular specimen of annealed low density polyethylene was further investigated by comparison with its frequency dispersion behavior of dynamic crystal orientation. The mechanical and birefringence dispersions were found to be definitely associated with the dynamic crystal orientation and could be assigned to an $\alpha_{1}$ mechanism arising from the so-called grain boundary phenomena, but not to an $\alpha_{2}$ mechanism resulting from a crystal disordering transition or pre-melting.

The temperature dependence of the vertical shift factor for bringing the mechanical data to its master curve, $b_{T}\left(T, T_{0}\right)$, was discussed in terms of Nagamatsu-Takemura's proposal, which takes into account the volume effect of the crystalline region, and of Wada-Okano's proposal, which takes into account the smearing out effect of the crystal lattice potential. Neither of the proposals gave any definite explanation of the temperature dependence nor any assignment of the mechanical dispersion. The temperature dependence of the vertical shift factor for optical data, $p_{T}\left(T, T_{0}\right)$, was also discussed on the basis of a two-phase hypothesis, i.e., additivity of crystalline and noncrystalline optical contributions, with the conclusion that the dependence results not only from that of the fractional volume of each phase but also from that of the orientation compliance of the noncrystalline chain segments for the particular specimen of annealed low density polyethylene.

KEY WORDS Dynamic Birefringence / Polyethylene / $\Pi$-Sector Technique / Crystalline Relaxation /
\end{abstract}

Numerous studies on the nature of the re-

\footnotetext{
* A part of the M.S. thesis of Thein Kyu presented to the Department of Polymer Chemistry, Faculty of Engineering, Kyoto University, on February 26th, 1974.

** Present address: Polymer Research Department, Central Research Organization, Kanbe, Yankin, Rangoon, Burma.

*** To whom correspondence should be addressed.
}

laxation mechanisms of polyethylene occurring in the so-called "crystalline dispersion region" in the dynamic modulus functions have reported that the dispersion ( $\alpha$ dispersion) consists of two or more relaxation mechanisms. The resolution of the $\alpha$ dispersion into two components was first investigated by Nakayasu, et al., ${ }^{1}$ for a melt-crystallized linear polyethylene; they were designated mechanisms I and II, with activation 
energies of the relaxation processes of 28 and $46 \mathrm{kcal} / \mathrm{mol}$, respectively. Since then, multiple types of crystalline relaxation mechanisms have been extensively studied by many authors, mostly in terms of the so-called "relaxation maps" from experimental sources of isochronal data of the temperature dependence of dynamic modulus functions of the polymers in bulkcrystallized as well as solution grown states. ${ }^{2-10}$ The speculated assignments for the crystalline relaxation mechanisms can be classified into two categories: i.e., a mechanism associated with the crystal disordering transition or premelting and one with the grain boundary phenomena $^{11)}$ arising from various types of crystal orientation and/or deformation in an amorphous medium.

Takayanagi has proposed two types of crystalline relaxation mechanisms, designated as $\beta_{\mathrm{c}}$ and $\alpha_{\mathrm{c}}$ mechanisms, which are associated with the crystal disordering transition due to the onset of different modes of molecular motions of polymer chains around their axes and translational vibration of the polymer chain segments along the chain axis, respectively. ${ }^{4-6,9} \mathrm{He}$ added later a mechanism, designated as $\alpha_{\mathrm{c}}{ }^{\prime}$, arising from friction between mosaic blocks within annealed polymer crystals, ${ }^{10}$ which may be classified as one of the grain boundary phenomena. The activation energies of the $\beta_{\mathrm{c}}, \alpha_{\mathrm{c}}{ }^{\prime}$, and $\alpha_{\mathrm{c}}$ mechanisms for linear polyethylene, for example, have been found to be 16,25 , and $42 \mathrm{kcal} / \mathrm{mol}$, respectively.

On the other hand, Iwayanagi has proposed two types of crystalline relaxation mechanisms, designated as $\alpha_{1}$ and $\alpha_{2},{ }^{12}$ which are associated with the grain boundary phenomena of deformation and/or rotation of crystallites (crystal mosaic blocks) within a viscous medium (the amorphous medium) and with the crystal disordering transition due to the onset of torsional oscillation of polymer chains within the crystal lattice, respectively. The activation energy of the $\alpha_{1}$ mechanism is, therefore, expected to be of the same order as that of the primary relaxation mechanism ( $\beta$ dispersion), whereas the activation energy of the $\alpha_{2}$ mechanism for polyethylene, for example, is expected from theoretical $^{13,14}$ and experimental (NMR and dielectric $)^{15-19}$ considerations to be several tens of $\mathrm{kcal} / \mathrm{mol}$.
Some mechanical and dielectric studies on the solution grown crystals of linear polyethylene have also assigned the crystalline dispersion to be composed of two components; one arises from reorientation of the chain folds and the other is related to the coupling effect of torsional or/and translational motions of chain molecules within the crystals. $8,9,17,20,21$ These are related to the grain boundary phenomena and the crystal disordering transition, respectively. The effect of solvents on the $\alpha$ dispersion of polyethylene has been investigated by Hideshima, et al., ${ }^{22}$ who indicated a definite effect on the lower temperature dispersion corresponding to the $\alpha_{1}$ mechanism but little effect on the higher temperature dispersion corresponding to the $\alpha_{2}$ mechanism, and, consequently, suggested the $\alpha_{1}$ mechanism to be associated with the grain boundary phenomena involving the molecular motions in the surface of crystal lamellae.

Strictly speaking, however, the above assignments for the so-called crystalline relaxation processes are still based on considerable speculation except for relatively definite, though still indirect, evidence for the $\alpha_{2}$ mechanism. The recent results of rheo-optical investigations using the dynamic X-ray diffraction technique ${ }^{23,24}$ for dynamic orientation and lattice deformation of polyethylene crystals, ${ }^{25-27}$ as well as the dynamic light-scattering technique ${ }^{28,29}$ for the deformation mechanism of polyethylene spherulites, ${ }^{30,31}$ provide the most definitive evidence to confirm that the so-called "crystalline relaxation processes" are really related to the crystalline responses.

In this paper, the dynamic birefringence behavior of low- and medium-density polyethylenes, which is obtained by means of the $\pi$-sector technique described in Part I of this series, ${ }^{32}$ will be presented in order to discuss the nature of the crystalline relaxation processes in more detail for a particular specimen of the polyethylenes whose dynamic X-ray diffraction behavior has been observed in this laboratory. The dynamic birefringence is a sensitive optical quantity for detecting the dynamic orientation of polymer molecules, as a whole, but is not necessarily a quantity which is able to distinguish the crystalline and noncrystalline responses. 
In turn, however, a combination of the dynamic birefringence behavior with the dynamic X-ray diffraction behavior will enable us to investigate the noncrystalline responses in the semicrystalline materials separately and to assign the $\alpha$ dispersion in terms of more definitive structural responses.

\section{TEST SPECIMENS AND EXPERIMENTAL PROCEDURES}

Test polymers used in this experiment are a low-density polyethylene (Sumikathene G201, Sumitomo Chemical Co.) and a medium-density polyethylene (Sumikathene KP119, Sumitimo Chemical Co.) having viscosity-average molecular weights of 42,000 and 170,000 and degrees of branching of 2.5 and $1.3 \mathrm{CH}_{3} / 100 \mathrm{C}$, respectivery. Film specimens of the low- and medium-density polyethylenes were prepared by placing the pellets in an aluminum spacer sandwiched in between two stainless steel plates and melting at 140 and $160^{\circ} \mathrm{C}$, respectively, for about $10 \mathrm{~min}$ in a laboratory press. A pressure of $200 \mathrm{~kg} / \mathrm{cm}^{2}$ was applied for $10 \mathrm{~min}$ at both molten states at 140 and $160^{\circ} \mathrm{C}$, and the molten temperatures were slowly cooled down to 105 and $115^{\circ} \mathrm{C}$ for moulding the film specimens of the low and medium density polyethylenes, respectively. The film specimens formed between the stainless steel plates were then plunged into ice water together with the plates and are designated as quenched specimens, G201(Q) and KP119(Q). The quenched films thus obtained were annealed at $95^{\circ} \mathrm{C}$ in a vacuum oven at free length for $2 \mathrm{hr}$ and are designated as annealed specimens, G201(A) and KP119(A). Additional specimens with a different thermal history from the above, were prepared by allowing the moulded films to remain at 105 and $115^{\circ} \mathrm{C}$ for $2 \mathrm{hr}$ after being slowly cooled from the molten states, then further slowly cooled down to room temperature. These specimens are designated as slowly cooled specimens, G201(H) and $\mathrm{KP} 119(\mathrm{H})$.

The thickness of the film specimens was adjusted by an aluminum spacer so as to fit the dynamic birefringence measurements, i.e., about $140 \mu$ for the medium-density polyethlene, which is two times thinner than that of the low-density
G20I

$(Q)$

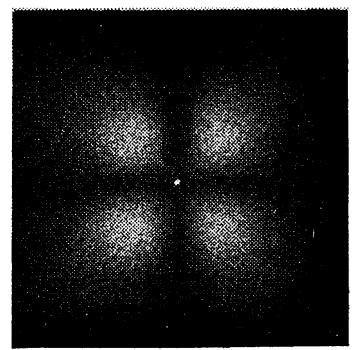

KPII9

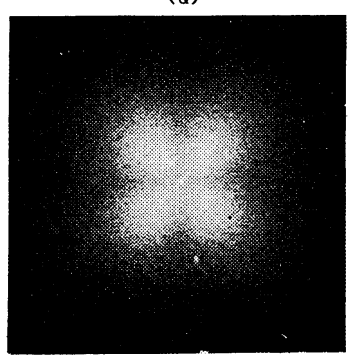

(A)

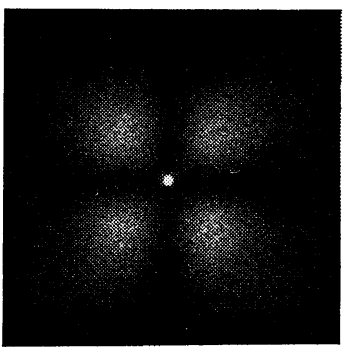

(A)

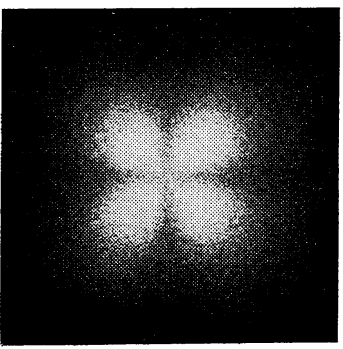

(H)

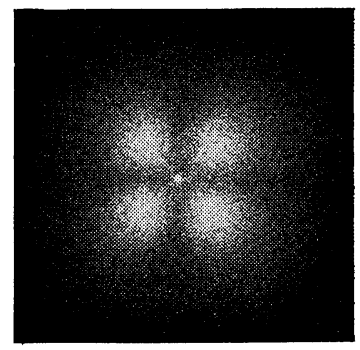

(H)

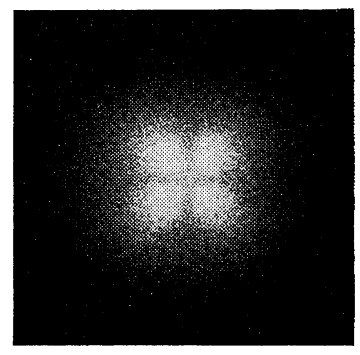

$0 \quad 5 \quad 10,15^{\circ}$

Figure 1. $H_{v}$ ligth scattering patterns of two kinds of polyethylene films, low- and mediumdensity polyethylene films, with different thermal histories. 
polyethylene films of more transparence. The densities of $\mathrm{G} 201(\mathrm{Q}), \mathrm{G} 201(\mathrm{~A})$, and $\mathrm{G} 201(\mathrm{H})$ were found to be $0.916,0.920$, and $0.922 \mathrm{~g} / \mathrm{cm}^{3}$, while those of KP119(Q), KP119(A), and $\mathrm{KP} 119(\mathrm{H})$ were $0.932,0.934$, and 0.937 , as evaluated from an 2-propanol-water density gradient tube at $30.0^{\circ} \mathrm{C}$. Hence, it can be noted that the thermal history of the specimens does not appreciably influence the density, i.e., the degree of crystallinity. These film specimens were used in the polarized light-scattering study, which confirmed that all specimens contain a spherulitic crystalline texture ranging up to about $10 \mu$ in size. The spherulites of the slowly cooled specimens are somewhat larger in size and more perfect in spherulitic texture than those of the quenched and annealed specimens, as recognized from the $\mathrm{H}_{\mathrm{v}}$ light-scattering patterns in Figure 1.

These film specimens were kept in a dessicater for about a week before being used in the dynamic measurements in order to prevent any absorption of moisture and to allow the specimens to reach a more stable state. The film specimen is cut into a ribbon shape, $1 \mathrm{~cm}$ wide and $8 \mathrm{~cm}$ long, clamped initially at a gauge length of $6 \mathrm{~cm}$, and is subjected to $3.3-\%$ static tensile strain superposed with a dynamic tensile strain of $0.25 \%$. A preparatory vibration is applied for about $1 \mathrm{hr}$ at a frequency of $4.3 \mathrm{~Hz}$ in order to assure the vibrational steady state as well as a sort of mechanical conditioning of the specimen. Then, the dynamic birefringence and mechanical measurements were carried out over a frequency range from 0.008 to $4.3 \mathrm{~Hz}$ at various temperatures from 30 to $90^{\circ} \mathrm{C}$.

\section{EXPERIMENTAL RESULTS AND DISCUSSION}

Mechanical Dispersion. As has been briefly reported in Part I of this series ${ }^{32}$ and is illustrated in Figures 2 and 3 for $\mathrm{G} 201(\mathrm{H})$, for typical examples, the storage modulus function $E^{\prime}(\nu)$ as well as the loss modulus function $E^{\prime \prime}(\nu)$ of all of the test specimens decrease remarkably with increasing temperature. The decrease produces the difficulty that the conventional frequency-temperature superposition simply by a horizontal shift of the modulus functions along the logarithmic frequency axis is invalid, and

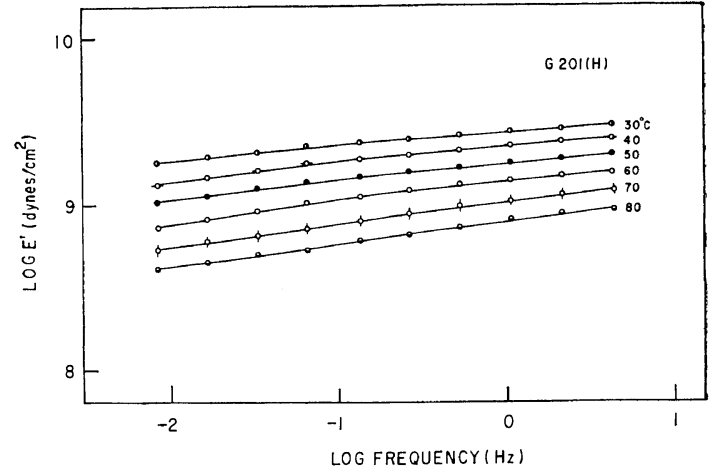

Figure 2. Temperature dependence of the storage modulus function of slowly cooled low-density polyethylene.

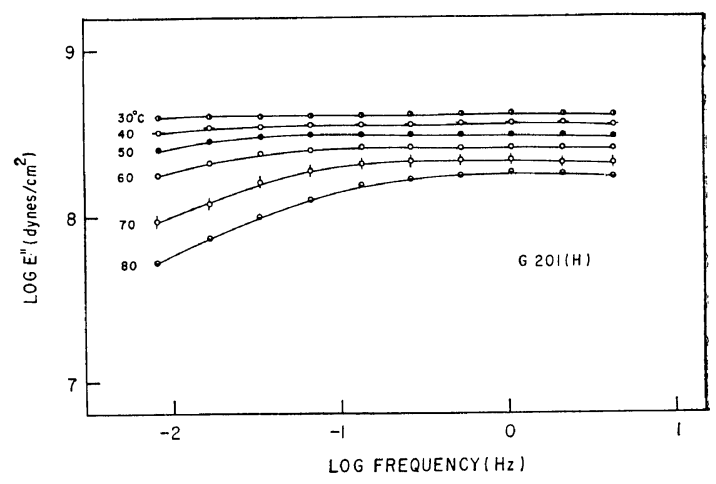

Figure 3. Temperature dependence of the loss modulus function of slowly cooled low-density polyethylene.

leads to the requirement of a considerable vertical shift of the functions in order to obtain good superposed master curves of the functions at a given reference temperature.

The proper vertical shift in the superposition is performed after the horizontal shift of the modulus functions by using the shift factors obtained from the horizontal shift of the mechanical loss angle tangent function, $\tan \delta(\nu)$. That is, the time-temperature superposition for a system composed of multiple types of relaxation mechanisms may be formulated as follows: ${ }^{33}$

$$
\begin{aligned}
E_{T}{ }^{\prime}(\nu) & =\sum_{j=1}^{m}\left(\rho / \rho_{0}\right) f_{j}\left(T, T_{0}\right) E_{j, T_{0}}^{\prime}\left(\nu a_{T_{j}}\right) \\
E_{T}{ }^{\prime \prime}(\nu) & =\sum_{j=1}^{m}\left(\rho / \rho_{0}\right) f_{j}\left(T, T_{0}\right) E_{j, T_{0}}^{\prime \prime}\left(\nu a_{T_{j}}\right)
\end{aligned}
$$

where $a_{T_{j}}$ is the horizontal shift factor for the 
$j$ th relaxation mechanism, $\rho_{0}$ is the density of the system at the reference temperature $T_{0}$, and $\left(\rho / \rho_{0}\right)$ is a correction factor due to the thermal expansion of the system. $f_{j}\left(T, T_{0}\right)$ is also a correction factor arising from the temperature dependence of the elastic moduli of the relaxation elements, $E_{i, j}(T)$, composing the $j$ th relaxation mechanism in the fashion of a generalized Maxwell model, and has been conventionally taken as $\left(T / T_{0}\right)$ for a relaxation mechanism having entropic elasticity. $\left(\rho / \rho_{0}\right) f_{j}\left(T, T_{0}\right)$ must be thus understood as related to the vertical shift factor $b_{T_{j}}$ for the $j$ th relaxation mechanism, phenomenologically.

If the observed modulus functions, $E_{T}{ }^{\prime}(\nu)$ and $E_{T}{ }^{\prime \prime}(\nu)$, could be well separated into the contribution from the $j$ th mechanism and those from the other mechanisms, $\tan \delta_{j, T}(\nu)$ could be written only in terms of $a_{T_{j}}$, and not of $b_{T_{j}}$, as follows:

$$
\tan \delta_{j, T}(\nu)=E_{j, T}^{\prime \prime}(\nu) / E_{j, T}^{\prime}(\nu)=\tan \delta_{j, T_{0}}\left(\nu a_{T_{j}}\right)
$$

Utilization of eq 3 will be a problem, however, unless either the separation of the $j$ th contri-

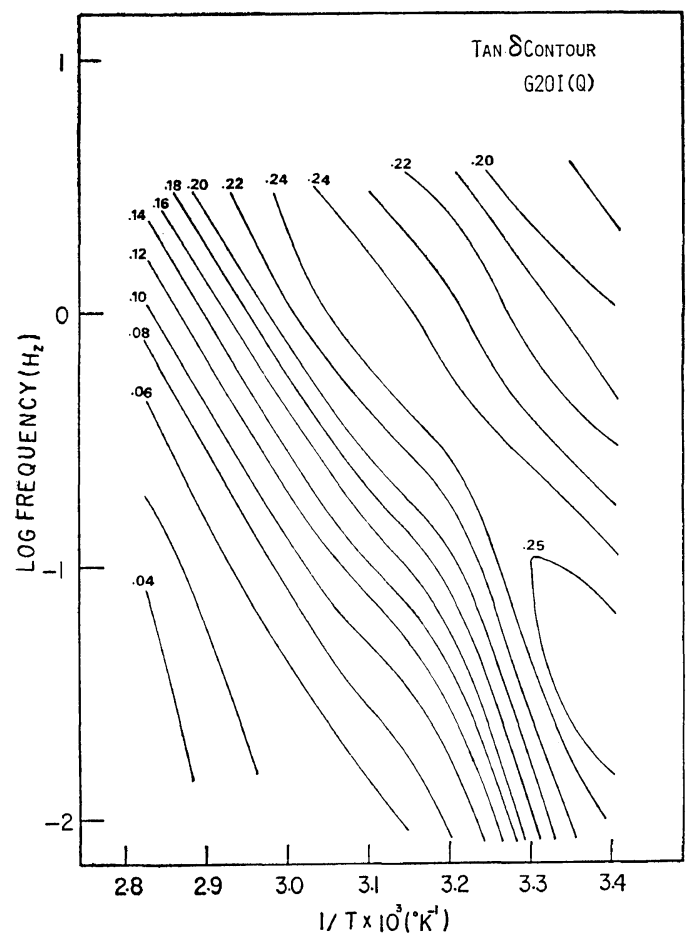

Figure 4. Tan $\delta$ contour map of quenched lowdensity polyethylene. bution from the others can be performed experimentally or the modulus functions, $E_{T}{ }^{\prime}(\nu)$ and $E_{T}{ }^{\prime \prime}(\nu)$, and, consequently, $\tan \delta_{T}(\nu)$ can be observed as isolated for the $j$ th relaxation mechanism.

Figures 4 and 5 show the so-called $\tan \delta$ contour maps which plot the observed $\tan \delta$ function against both temperature and frequency for $G 201(\mathrm{Q})$ and $\mathrm{G} 201(\mathrm{H})$, for example. As can be seen in the figures, the ridge is rather monotonous in height and the contour lines on both sides of the ridge are guite parallel to the ridge. This fact suggests that the observed $\tan \delta$ function is fairly well isolated for the $j$ th (crystalline) relaxation mechanism, and validates the estimation of $a_{T_{j}}$ by using eq 3 which is taken to be independent of $b_{T_{j}}$ with relatively small arbitrariness. ${ }^{*}$

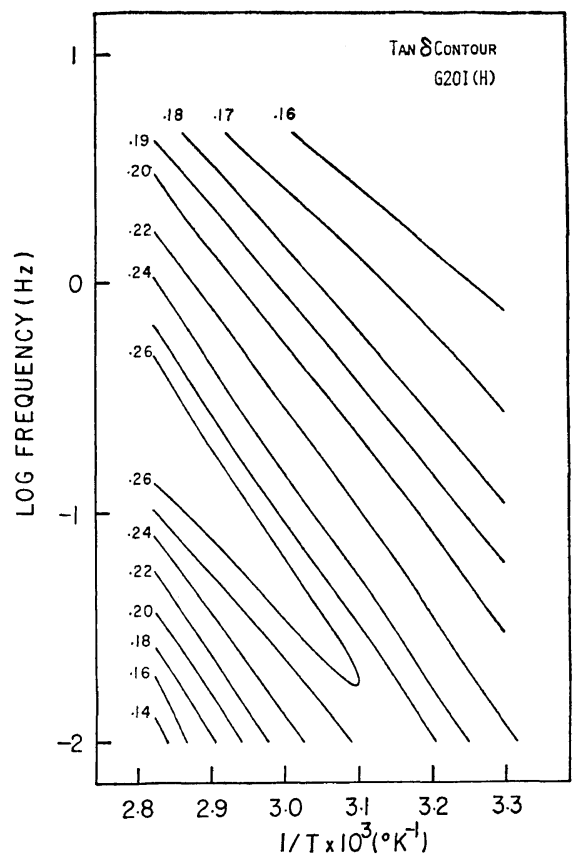

Figure 5. Tan $\delta$ contour map of slowly cooled low-density polyethylene.

* Strictly speaking, however, the ridge is not necessarily even in height; it decreases with increasing frequency and temperature for G201(Q) and vice versa for $\mathrm{G} 201(\mathrm{H})$, and the contour lines are not perfectly parallel to each other with equal contour intervals. These facts suggest that the isolation of the $j$ th relaxation mechanism is not sufficient enough, and is probably affected, more or less, by the contributions from the other relaxation mechanisms. 

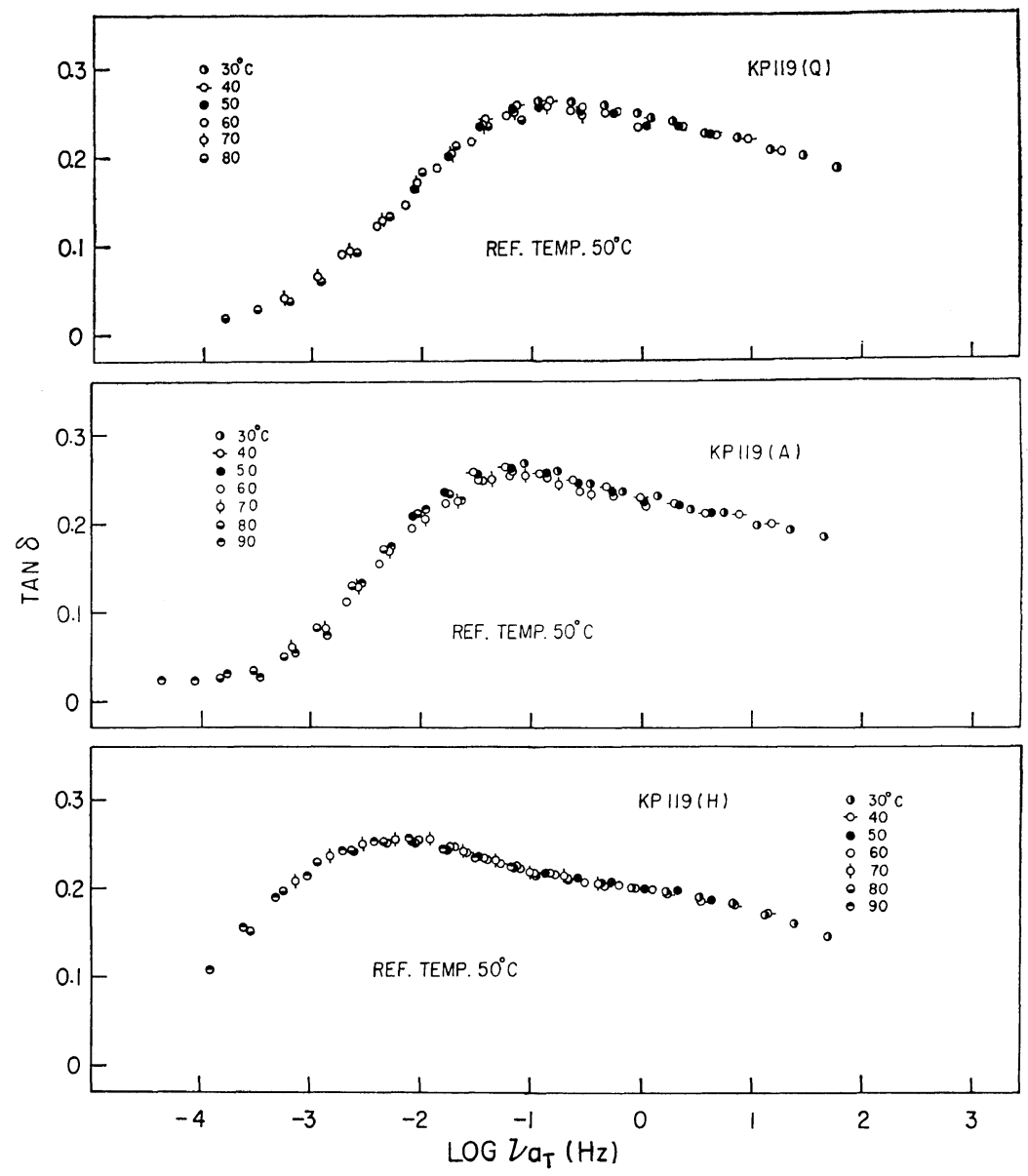

Figure 6. Master curves of $\tan \delta$ functions of a series of medium-density polyethylenes reduced to a given reference temperature of $50^{\circ} \mathrm{C}$.

Figure 6 shows a series of master curves of $\tan \delta_{j, T_{0}}\left(\nu a_{T_{j}}\right)$ for the KP119 specimens, all reduced to a given reference temperature of $50^{\circ} \mathrm{C}$. It can be seen that each master curve exhibits a distinct peak, which is not symmetric in form with respect to the reduced logarithmic frequency, and that the peak frequency shifts towards lower frequencies with progress of the heat treatment from $\mathrm{Q}$ to $\mathrm{A}$ to $\mathrm{H}$. Figure 7 shows a series of master curves of the loss modulus function, $b_{T_{j}} E_{j, T_{0}}^{\prime \prime}\left(\nu a_{T_{j}}\right)$, also for the KP119 specimens, all reduced to the reference temperature of $50^{\circ} \mathrm{C}$ by means of the above mentioned procedure which applies the vertical shift as well as the horizontal shift of the observed functions at various temperatures. It is seen that the frequency dispersion in the reduced master curve is quite broad, exhibiting no distinct peak, and shifts towards lower frequencies with the progress of the heat treatment. Such a broad dispersion without any distinct peak may be expected whenever there exists more than a single contribution mechanism in the relaxation. In addition, the shape of the reduced master curves is again asymmetric with respect to the reduced logarithmic frequency. Hence, if one assumes the contribution of each relaxation mechanism to the loss modulus function to be represented in terms of a symmetric functional form, such as the Gaussian functional form, ${ }^{33}$ the deviating portion at higher frequencies may be attributed to the contribution 
Dynamic Birefringence of Semicrystalline Polymers

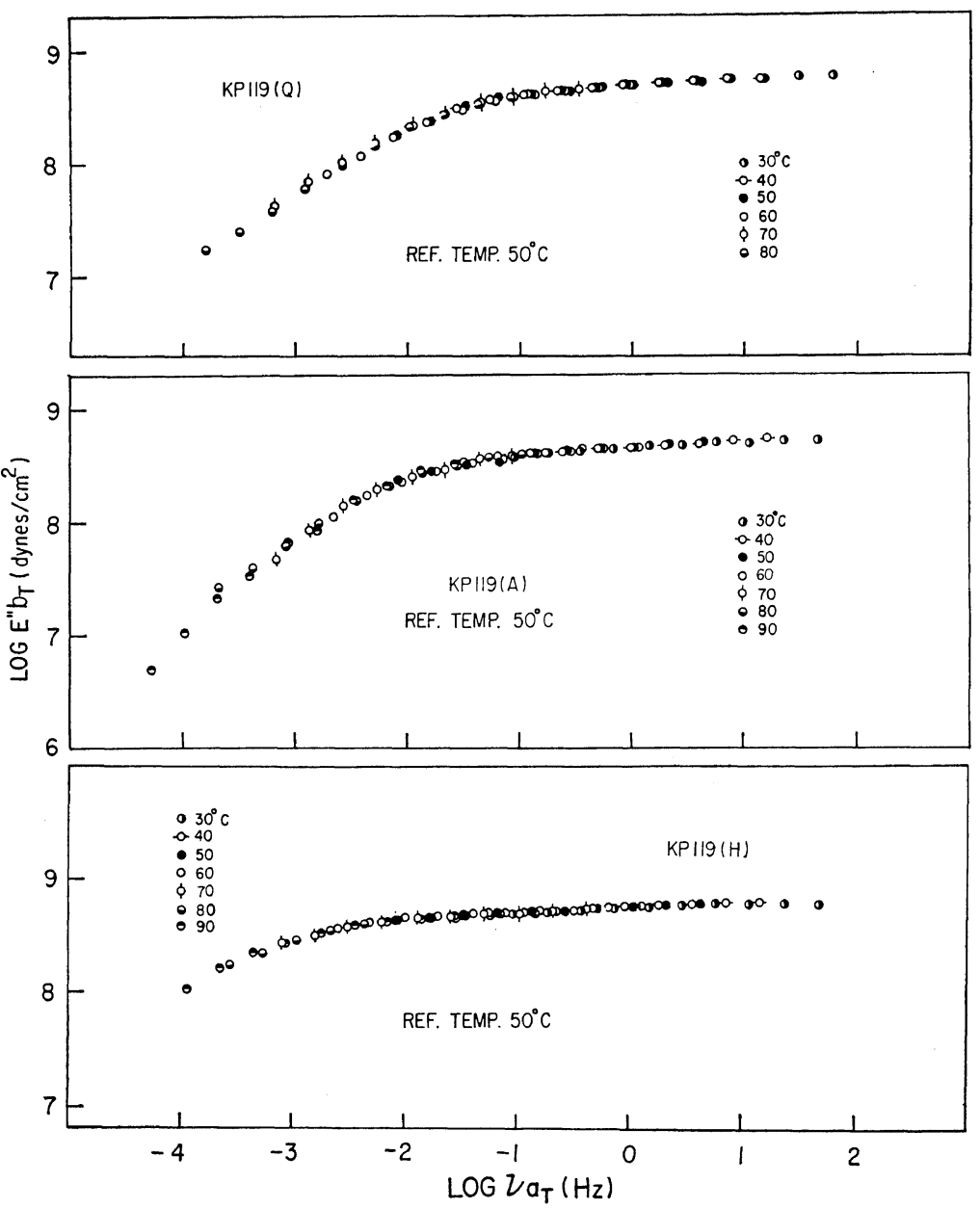

Figure 7. Master curves of the loss modulus functions of a series of medium-density polyethylenes reduced to a given reference temperature of $50^{\circ} \mathrm{C}$.

from the $(j-1)$ th relaxation mechanism, corresponding probably to the $\beta$ dispersion, while the deviating portion at lower frequencies, if any, may be connected with the contribution from the $(j+1)$ th mechanism arising from the double components of the $\alpha$ dispersion. At present, however, the separation of the reduced loss modulus function into the respective contributions, which is quite important to understand the full nature of the mechanisms, cannot be carried out due to the lack of adequate data, especially at even higher frequencies and lower temperatures, for the $\beta$ dispersion.

The general behavior of the reduced master curves of $\tan \delta$ and loss modulus functions for the G201 specimens is similar to that for the KP119 specimens just mentioned above, expect for the fact that the $\tan \delta$ peak frequencies and, consequently, the frequency dispersion in the loss modulus function, both reduced to the same reference temperature of $50^{\circ} \mathrm{C}$, appear at higher reduced frequencies than those for the corresponding specimens of KP119.

Figure 8 shows the temperature dependences of the horizontal shift factors $a_{T}\left(T, T_{0}\right)$, which are obtained from $\tan \delta_{T}(\nu)$ in order to compose the master curve of $\tan \delta_{j, T_{0}}\left(\nu a_{T_{j}}\right)$ and are plotted in terms of logarithmic value against reciprocal of absolute temperature. As can be seen in the figure, each temperature dependence is 


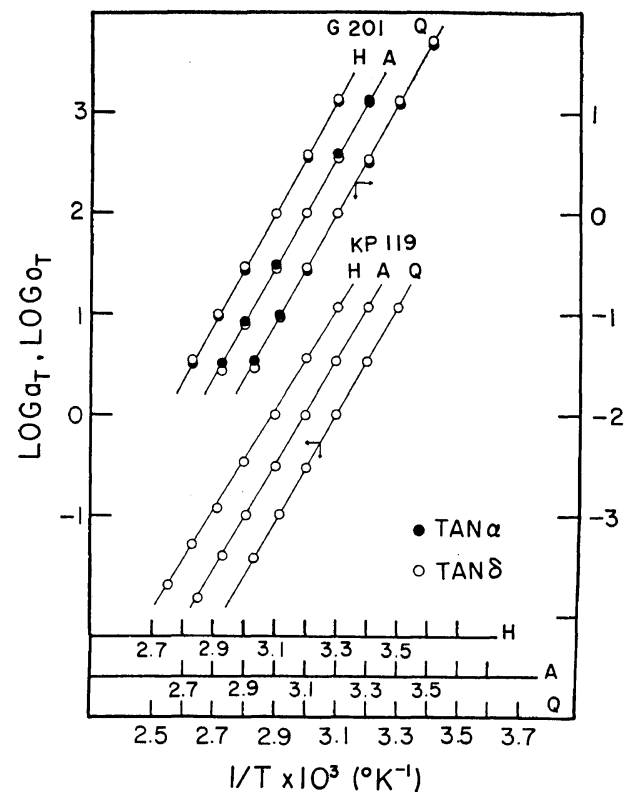

Figure 8. Temperature dependence of the horizontal shift factor $a_{T}\left(T, T_{0}\right)$ for constructing master curves of $\tan \delta$ functions of two series of low- and medium-density polyethylenes, taking the reference temperature as $50^{\circ} \mathrm{C}$. That of horizontal shift factor $o_{T}\left(T, T_{0}\right)$ for constructing master curves of complex dynamic strain-optical coefficients, $K^{*}$ of a series of low density polyethylenes is also plotted.

represented by a straight line, showing the Arrhenius type of temperature dependence, at least over the temperature range covered, irrespective of the kind of polymer and of the type of heat treatment. The slope of the straight line is a little steeper for the medium density polyethylene and becomes a little less steep with the progress of the heat treatment, giving activation energies of the $j$ th relaxation process ranging from 25 to $27 \mathrm{kcal} / \mathrm{mol}$; these are quite consistent with the literature values of the $\alpha_{1}$ mechanism. ${ }^{1,3,7,10,22}$

Figure 9 shows the temperature dependences of the vertical shift factors $b_{T}\left(T, T_{0}\right)$, which are obtained by constructing the master curve of the loss modulus function $b_{T_{j}} E_{j, T_{0}}^{\prime \prime}\left(\nu a_{T_{j}}\right)$ after the horizontal shift of $E_{T}^{\prime \prime}(\nu)$ using $a_{T_{j}}$. As can be seen in the figure, the temperature dependence of $b_{T_{j}}\left(T, T_{0}\right)$ is given by a common straight line, irrespective of the type of heat treatment, when reduced to the reference tem-

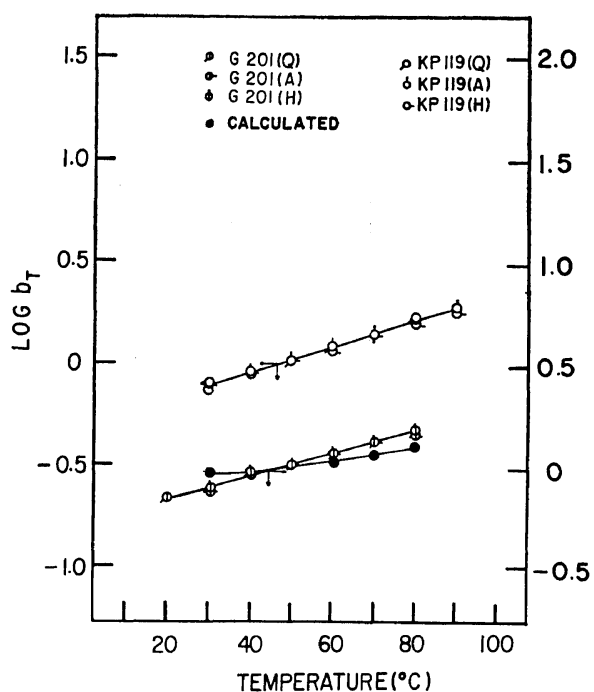

Figure 9. Temperature dependence of the vertical shift factor $b_{T}\left(T, T_{0}\right)$ for constructing master curves of loss modulus functions of two series of low- and medium-density polyethylenes, taking the reference temperature as $50^{\circ} \mathrm{C}$. The calculated dependence using Nagamatsu-Takemura's proposal which takes into account the volume effect of crystalline region is also plotted for annealed low density polyethylene.

perature of $50^{\circ} \mathrm{C}$ and plotted in logarithmic value against linear scale of temperature, though the slope of the straight line is somewhat steeper for the medium density polyethylene than for the low density polyethylene, $0.00253 / \mathrm{deg}$ and $0.00243 / \mathrm{deg}$ respectively.

The time-temperature superposition of the storage modulus function $E_{T}{ }^{\prime}(\nu)$ by means of the above mentioned procedure using the horizontal and vertical shift factors, $a_{T_{j}}\left(T, T_{0}\right)$ and $b_{T_{j}}\left(T, T_{0}\right)$, which are obtained from the $\tan \delta$ and loss modulus functions, is quite satisfactory in general; these results are not shown to save space. The facts that the temperature dependence of the horizontal shift factor is given by a single Arrhenius type equation and that the activation energy of the relaxation process is found to be near the literature values proposed by several authors for the $\alpha_{1}$ mechanism, irrespective of both the kind of polymer and of the type of heat treatment, may indicate that the observed mechanical behavior is associated 
mainly with the isolated $j$ th relaxation mechanism.

Significance of the Vertical Shift Factor $b_{T}$. The temperature dependence of the vertical shift factor for polyethylene has been discussed by Nagamatsu, et al., in relation to that of the degree of crystallinity, ${ }^{34}$ based on a model proposed by Takemura. ${ }^{35}$ The temperature dependence of $b_{T}$ for a low-density polyethylene, Alkathene, is also given by a straight line, when plotted in logarithmic value against linear scale of temperature ranging from 0 to $80^{\circ} \mathrm{C},{ }^{34}$ though the slope of the straight line is about three times steeper than those obtained here.

Actually, the formulation of the temperature dependence of $b_{T}$ from Takemura's model, i.e., $f_{j}\left(T, T_{0}\right)$ in eq 1 and 2 in the present paper, is based primarily on the temperature dependence of noncrystalline entropic elasticity which is supplimented in terms of the volume effect of crystalline region, i.e., the temperature dependence of the degree of crystallinity, but not substantially on that of the associated elasticity itself. Therefore, the formulation may be accepted for the $\beta$ dispersion rather than the crystalline dispersion, unless a considerable modification of the noncrystalline entropic elasticity is made so as to reflect the grain boundary phenomena for the $\alpha_{1}$ mechanism. The dots in Figure 9 show calculated results for G201(A), for example, from the formulation which takes into account the temperature dependence of Xray crystallinity shown in Figure 18. The agreement with the experimental results is not necessarily satisfactory.

Hence, the mechanical relaxation at relatively high temperatures may be expressed by the following equations, as suggested by Kaneko, et $a l .,{ }^{36,37}$

$$
\begin{aligned}
& E_{j, T}(t)=E_{j, T_{0}}\left(t / a_{T_{j}}\right) \exp \left[-\beta^{\prime}\left(T-T_{0}\right)\right] \\
& E_{j, T}^{*}(\nu)=E_{j, T_{0}}^{*}\left(\nu a_{T_{j}}\right) \exp \left[-\beta^{\prime}\left(T-T_{0}\right)\right]
\end{aligned}
$$

where $\beta^{\prime}$ corresponds to the slope of the logarithmic vertical shift factor $b_{T}$ plotted against temperature as shown in Figure 9. Let us check the temperature dependence of $b_{T}$, i.e., $\beta^{\prime}$, in a different way by taking into account the temperature dependence of the crystalline elasticity.

Wada et al., ${ }^{13,15,38}$ and Okano ${ }^{14}$ have pro- posed that the crystalline dispersion occurs because of the incoherent lattice vibration within crystals in which the intermolecular potential suffers a smearing-out effect. Peterlin et al. ${ }^{39-41}$ have treated the smearing-out effect as arising from either longitudinal or torsional lattice vibrations and have approximated the temperature dependence of the potential as follows:

or

$$
\Phi_{T}=\Phi_{\mathrm{c}} \exp \left[-\pi^{2} N k T /\left(5 f a^{2}\right)\right]
$$

$$
\Phi_{T}=\Phi_{\mathrm{c}} \exp \left[-N k T /\left(5 f^{\prime}\right)\right]
$$

where $\Phi_{T}$ is the intermolecular potential at temperature $T$ with the smearing-out effect, $\Phi_{\mathrm{c}}$ the intermolecular potential in the absence of the effect, $N$ the number of atomic units along the crystalline chain axis, a the atomic distance between neighboring atoms along the chain axis, and $f$ and $f^{\prime}$ the longitudinal and torsional force constants, respectively.

Assuming that the temperature dependence of the elastic moduli of the relaxation elements $E_{i, j}(T)$ which make up the $j$ th relaxation mechanism suffer in equal magnitude the smearing-out effect, then $\beta^{\prime}$ in eq 4 and 5 can be rewritten, as given by Kaneko, et al., ${ }^{36}$ in terms of either type of the smearing-out effects, as follows:

or

$$
\beta^{\prime}=\left[\pi^{2} N k /\left(5 f a^{2}\right)\right]
$$

$$
\beta^{\prime}=\left[N k /\left(5 f^{\prime}\right)\right]
$$

If we take $a=1.25 \mathrm{~A}$ and $N=70$ and 85 for the low and medium density polyethylenes on the average, which are estimated from the long spacing obtained from X-ray small angle scattering for both kinds of polyethylenes as $190 \pm$ $20 \mathrm{~A}$, and from the volume fraction of the crystalline region from the density values on the average for the two kinds of polyethylenes as 45 and $55 \%$, and $\beta^{\prime}=0.00253$ and 0.00243 , then the force constants, $f$ and $f^{\prime}$, are found from eq 8 and 9 to be $0.5 \times 10^{5}$ and $0.6 \times 10^{5}$ $\mathrm{dyn} / \mathrm{cm}$ per bond and as $0.8 \times 10^{-12}$ and $1.0 \times$ $10^{-12}$ erg for the low and medium density polyethylenes, respectively. These values of $f$ are considerably smaller than those proposed by Kuhn and $\mathrm{Kuhn}^{42}$ and Shimanouchi, et al., ${ }^{43}$ $2.0 \times 10^{5}$ and $4.9 \times 10^{5} \mathrm{dyn} / \mathrm{cm}$ per bond, respectively, while the values of $f^{\prime}$ are rather in close agreement with those proposed by $\mathrm{Okano}^{14}$ and 
Table I. Values of $\beta^{\prime}$ as evaluated by using four sets of $f$ and $f^{\prime}$ with different probabilities for the smearing-out effects arising from longitudinal and torsional lattice vibrations within polymer crystals

\begin{tabular}{ccccc}
\hline \multirow{2}{*}{$p_{\mathrm{t}} /\left(p_{1}+p_{\mathrm{t}}\right)$} & Set I & Set II & Set III & Set IV \\
\cline { 2 - 5 } & $\begin{array}{c}f=4.9 \times 10^{5} \mathrm{dyn} / \mathrm{cm} \\
f^{\prime}=1.1 \times 10^{-12} \mathrm{erg}\end{array}$ & $\begin{array}{c}f=2.0 \times 10^{5} \\
f^{\prime}=1.1 \times 10^{-12}\end{array}$ & $\begin{array}{c}f=4.9 \times 10^{5} \\
f^{\prime}=0.5 \times 10^{-12}\end{array}$ & $\begin{array}{c}f=2.0 \times 10^{5} \\
f^{\prime}=0.5 \times 10^{-12}\end{array}$ \\
\hline 0 & $0.00055 / \mathrm{deg}$ & 0.00134 & 0.00055 & 0.00134 \\
0.5 & 0.00223 & 0.00262 & 0.00125 & 0.00170 \\
1 & 0.00390 & 0.00390 & 0.00195 & 0.00195 \\
\hline
\end{tabular}

Szigeti, ${ }^{44} 1.1 \times 10^{-12}$ and $0.5 \times 10^{-12} \mathrm{erg}$.

Table I lists four sets of calculated results of $\beta^{\prime}$ by using the force constants, $f$ and $f^{\prime}$ proposed by Kuhn and Kuhn, Shimanouchi, et al., Okano, and Szigeti, on the basis that the smearing-out effect arises from the longitudinal and torsional lattice vibrations, simultaneously, with probabilities of $p_{1}$ and $p_{\mathrm{t}}$ : i.e.,

$$
\beta^{\prime}=p_{1}\left[\pi^{2} N k /\left(5 f a^{2}\right)\right]+p_{t}\left[N k /\left(5 f^{\prime}\right)\right]
$$

where $p_{1}+p_{\mathrm{t}}=1$. As can be seen in the table, the results with equal probabilities, such as sets I and II, or the results with, at least, a greater contribution of the torsional lattice vibration than the longitudinal one give closer values of $\beta^{\prime}$ to those observed. In other words, the results with the longitudinal lattice vibration alone give too small values of $\beta^{\prime}$.

As briefly discussed above, neither of the theories proposed for explaining the temperature-dependence of the vertical shift factor $b_{T}$ in terms of the smearing-out effects of crystal potential can give any definite assignment of the relaxation process observed, except for a rather positive objection to the speculation about the crystalline relaxation mechanism due to the onset of the longitudinal lattice vibration alone. In addition, the value of the activation energy of the relaxation process, around $25 \mathrm{kcal} / \mathrm{mol}$, which is obtained from the temperature-dependence of the horizontal shift factor $a_{T}$ following an Arrhenius type equation, may be too small to assign the process to be the crystalline relaxation due to the onset of the torsional lattice vibration, coupled with the longitudinal lattice vibration with probability $p_{\mathrm{t}}$ at least greater than $(1 / 2)$, i.e., the so-called $\alpha_{2}$ mechanism.

Optical Dispersion. The optical results obtained simultaneously with the mechanical data are analyzed in terms of the real and imaginary parts of the strain optical coefficient, $K^{\prime}$ and $K^{\prime \prime}$, and the optical loss angle tangent, $\tan \alpha$, defined by $K^{\prime \prime} / K^{\prime}$. As has been pointed out in a previous $\operatorname{paper}^{32}$ and is illustrated in Figures 10 and 11 for G201(A) and KP119(A), respectively, both $K^{\prime}$ and $-K^{\prime \prime}$ show maxima within the temperature and frequency ranges covered. The maxima increase slightly with increasing temperature at relatively lower temperatures for some of the specimens, but generally decrease with increasing temperature with a much greater rate for $K^{\prime}$ than for $-K^{\prime \prime}$. The temperaturedependence of the optical coefficients on a given time scale shows, therefore, an inversion point of the dependence, which is consistent with those observed by other authors for polyethylenes, ${ }^{45-48}$ depending on the choice of the given time scale. The behavior is, however, considerably different in detail from those observed, for example by Takeuchi and Stein $^{47}$ for another kind of medium density polyethylene and by Onogi, et $a l^{46}$ for another kind of low density polyethylene; in both of these the time-temperature superposition has been performed only by using the horizontal shift of the data along the logarithmic time scale within a limited range of temperature up to $T_{\mathrm{m}}$, which approximately corresponds to the apparent inversion point of the temperature-dependence of the coefficients on a given time scale. In other words, the simple phenomenological findings of the horizontal shift factors from the $\tan \alpha_{T}(\nu)$ function and, subsequently, of the common vertical shift factors for $K^{\prime}$ and $-K^{\prime \prime}$, if any, on the bases of corresponding relations to eq 1 through 3 , seem to be quite invalid. These complications, which are more obvious 
Dynamic Birefringence of Semicrystalline Polymers

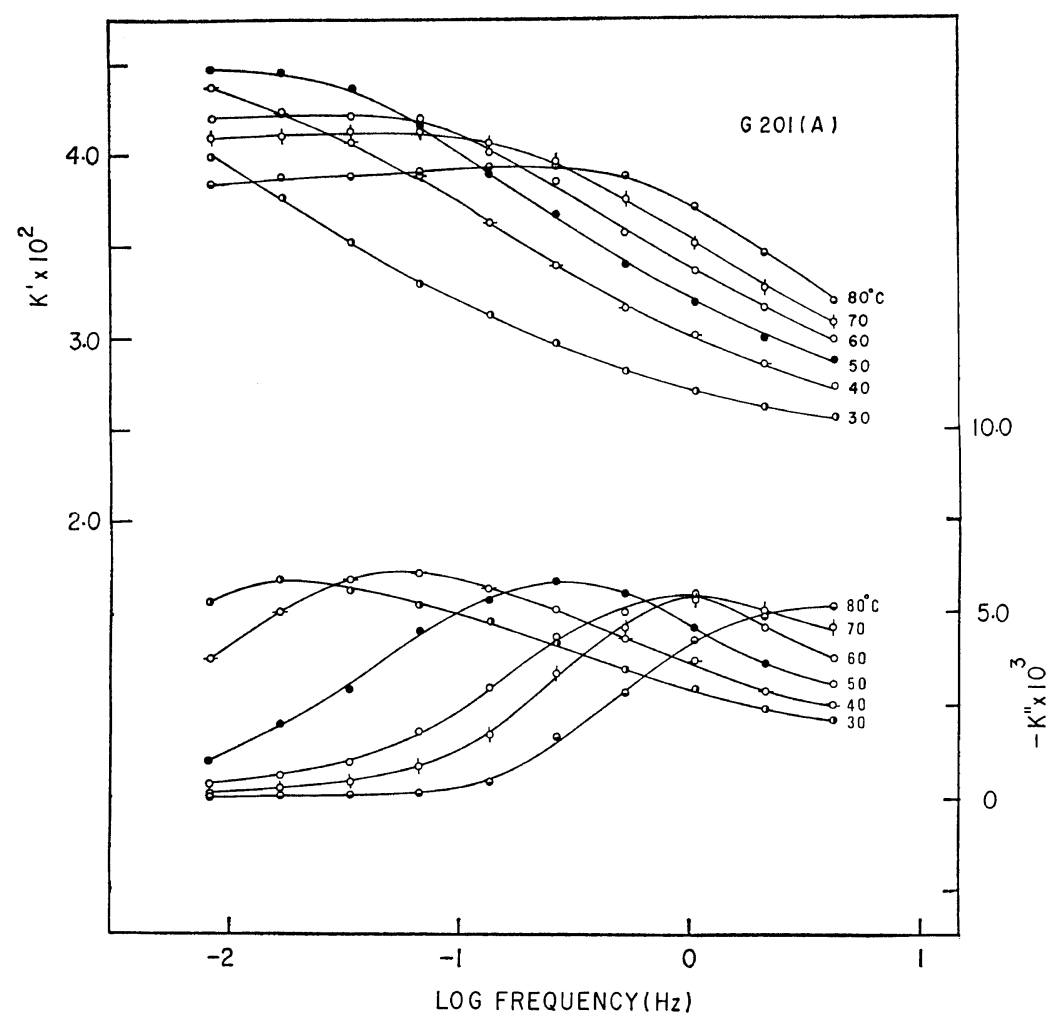

Figure 10. Temperature dependence of the real and imaginary parts of the complex dynamic strain-optical coefficient $K^{*}(i \nu)$ of annealed low-density polyethylene.

for the KP119 series than for the G201 series, must be understood in terms of the coexistence of at least two types of optical relaxation mechanisms contributing to the bulk responses, $K^{\prime}$ and $-K^{\prime \prime}$, to different extents.

Figures 12 and 13 show the so-called $\tan \alpha$ contour maps which plot the observed $\tan \alpha$ function against both temperature and frequency for G201(A) and KP119(A), respectively. Reflecting the above-mentioned complications, the contour map for $\mathrm{G} 201(\mathrm{~A})$ is rather simple, with a monotonous ridge line and almost parallel contour lines to the ridge, similar to the $\tan \delta$ maps in Figures 4 and 5, while that for KP119(A) is guite disturbed in its contour lines at relatively low temperatures and high frequencies, strongly suggesting an additional contribution from another optical relaxation mechanism than the $j$ th mechanism, i.e., the $(j-1)$ th mechanism.

The separation of the contribution of the $(j-1)$ th mechanism, if any, from the observed responses, $K^{\prime}$ and $-K^{\prime \prime}$, must be performed more rigorously than in the mechanical data, before the procedure of the time-temperature superposition. The separation has, however, been neglected due to the same reason as for the mechanical data, i.e., the lack of adequate data at even higher frequencies and lower temperatures where the data must be affected more seriously than the mechanical data by the $(j-1)$ th mechanism; the procedure of the timetemperature superposition is carried out by means of a conventional trial and error method with the restriction of finding a common horizontal shift factor $o_{T}$ between the data of $K_{T}{ }^{\prime}(\nu)$ and $-K_{T}^{\prime \prime}(\nu)$. Consequently, the vertical shift factor $p_{T}$, which is obviously different for $K^{\prime}$ and $-K^{\prime \prime}$, as well as the horizontal shift factor $o_{T}$ are more or less arbitrary and just apparently successful in the sense that they use an insufficient, actually no, separation of the $j$ th mechanism. 
T. Kyu, N. Yasuda, S. Suehiro, S. Nomura, and H. KawaI

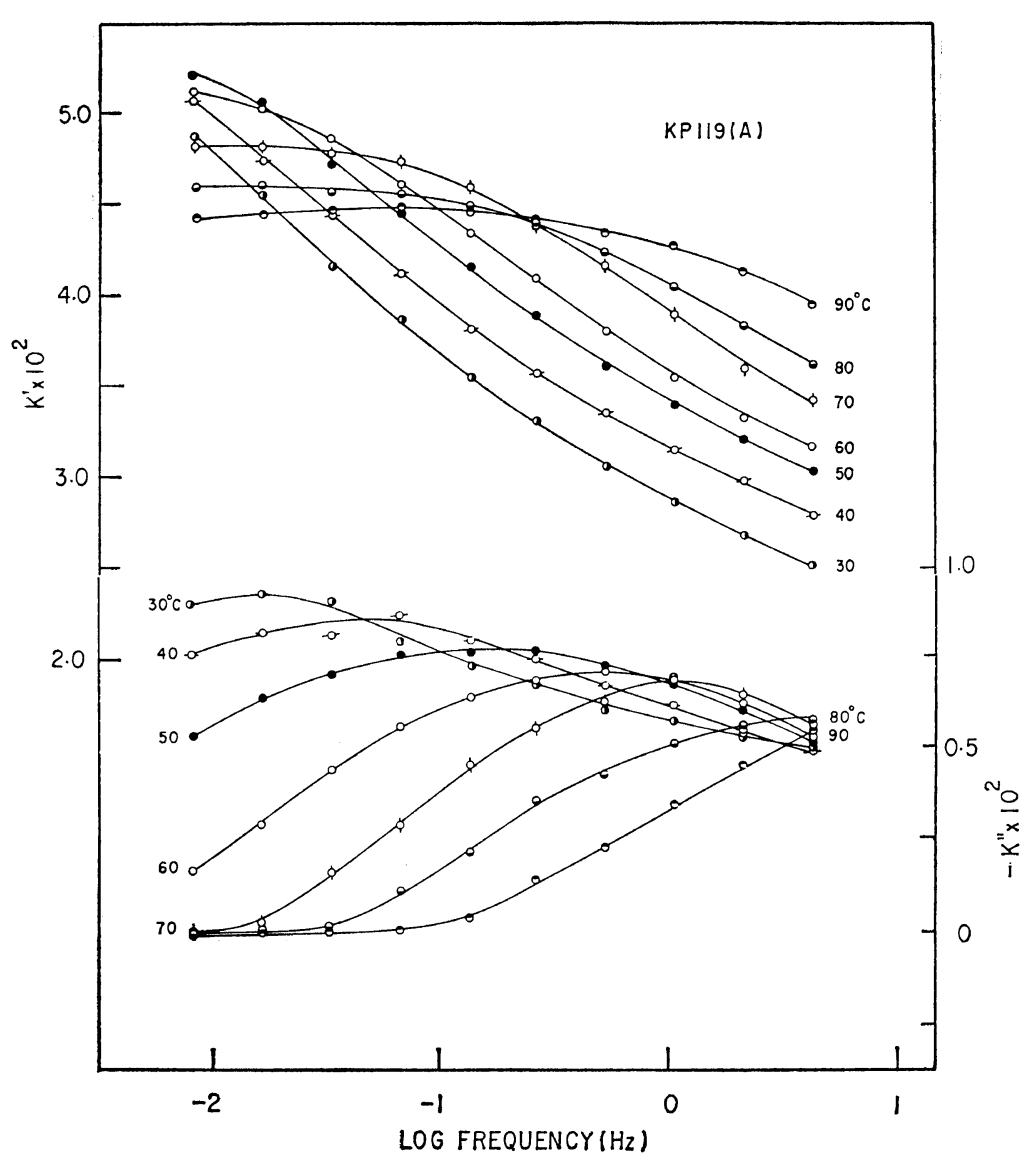

Figure 11. Temperature dependence of the real and imaginary parts of the complex dynamic strain-optical coefficient $K^{*}(i \nu)$ of annealed medium-density polyethylene.

Figures 14 and 15 show series of the apparent master curves of $K^{\prime}$ and $-K^{\prime \prime}$, i.e., $\left[K^{\prime}+\right.$ $\left.p_{T}\right]_{j, T_{0}}\left(\nu O_{T}\right)$ and $-\left[K^{\prime \prime}-p_{T}\right]_{j, T_{0}}\left(\nu O_{T}\right)$, for the KP119 specimens, respectively, all reduced to a reference temperature of $50^{\circ} \mathrm{C}$; here the subscript $j$ means the $j$ th mechanism in a much more tentative sense than the mechanical one, and the vertical shift factor $p_{T}$ is defined in a different fashion from $b_{T}$ because there is no guarantee that the optical coefficients keep the same sign over the ranges of temperature and frequency to be covered. As seen in the figures, every specimen shows definite optical dispersion over the reduced frequency range covered; this is characterized by a decrease of $\left(K^{\prime}+p_{T}\right)$ with increasing frequency and the appearance of a maximum in $-\left(K^{\prime \prime}-p_{T}\right)$. The frequency dis- persion shifts towards lower frequencies with progress of the heat treatment from $\mathbf{Q}$ to $A$ to $H$, similar to the mechanical dispersion in Figures 6 and 7, and is well-correlated to the mechanical dispersion, except for the fact that the $\tan \delta$ peak frequency appears at a slightly lower frequency than the $-\left(K^{\prime \prime}-p_{T}\right)$ peak frequency. The $-\left(K^{\prime \prime}-p_{T}\right)$ peak is asymmetric, similar to the $\tan \delta$ peak, with respect to the logarithmic reduced frequency; both show a shoulder on the higher frequency side. This shoulder must be related to the additional optical and mechanical relaxation mechanisms and should be assigned as the $(j-1)$ th mechanism. The general behavior of the apparent master curves of $\left(K^{\prime}+p_{T}\right)$ and $-\left(K^{\prime \prime}-p_{T}\right)$ for the G201 specimens is similar to that for the KP119 


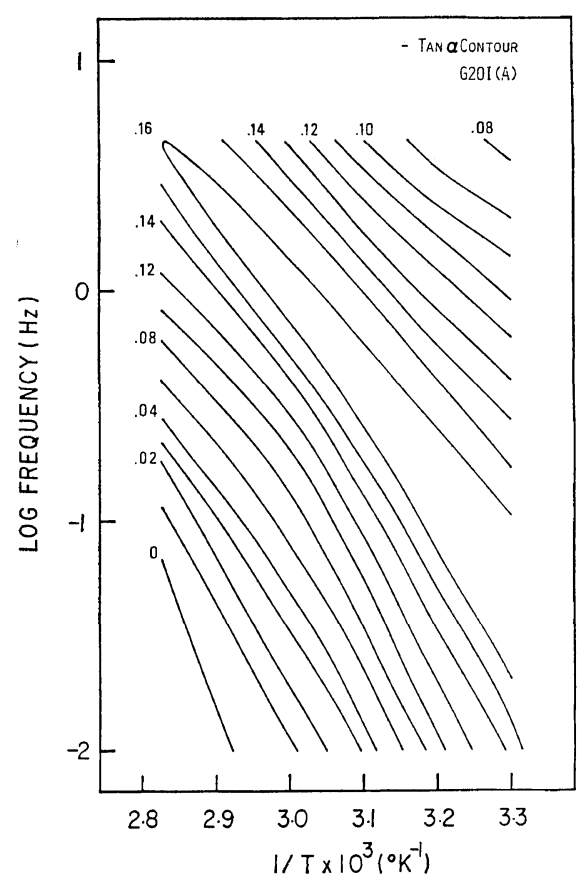

Figure 12. Tan $\alpha$ contour map of annealed lowdensity polyethylene.

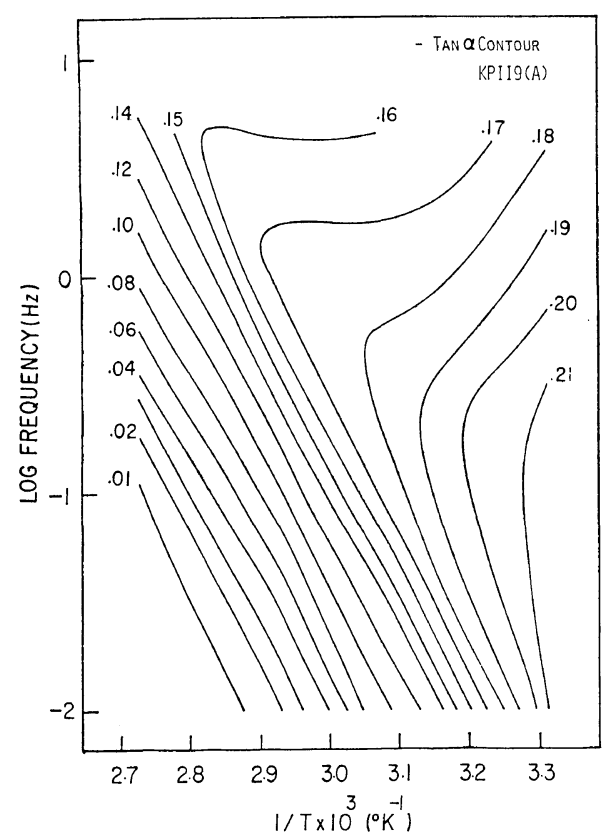

Figure 13. Tan $\alpha$ contour map of annealed mediumdensity polyethylene. specimens mentioned above, except for the fact that the frequency dispersion appears at a little higher reduced frequency, when reduced to the same reference temperature of $50^{\circ} \mathrm{C}$, than that for the corresponding specimens of KP119.

The temperature-dependence of the horizontal shift factor $o_{T}\left(T, T_{0}\right)$ for constructing the apparent master curves of the G201 specimens is found to coincide with that of $a_{T}\left(T, T_{0}\right)$, as plotted in Figure 8 with dot symbols, while that for the KP119 specimens differs from $a_{T}\left(T, T_{0}\right)$, as shown in Figure 16, giving two segments of straight line which bend at a particular temperature near $60^{\circ} \mathrm{C}$ to reveal two types of optical relaxation processes with activation energies of 21 to $23 \mathrm{kcal} / \mathrm{mol}$ and 33 $\mathrm{kcal} / \mathrm{mol}$ at the lower and higher temperature sides, respectively. Although the magnitudes of the activation energies may be too crude for any quantitative discussion due to the arbitrariness in determining $o_{T}\left(T, T_{0}\right)$, the two types of optical relaxation processes must be understood in terms of more discrete contributions of the $(j-1)$ th mechanism for the KP119 specimens than the G201 specimens and must be related to a more complicated $\tan \alpha$ map in Figure 13 for KP119(A) than that in Figure 12 for $\mathrm{G} 201(\mathrm{~A})$.

The temperature-dependence of the vertical shift factor $p_{T}\left(T, T_{0}\right)$ for constructing the apparent master curves, taking the reference temperature $T_{0}$ as $50^{\circ} \mathrm{C}$, is illustrated in Figure 17 for the two series of specimens. As can be seen in the figure, the dependence is, in general, much less obvious for $-K^{\prime \prime}$, than $K^{\prime}$, less obvious on the lower temperature side than the higher one, and less obvious with progress of the heat treatment, except for some anomalies for particular specimens. The change of the shift factor in sign from negative to positive at $50^{\circ} \mathrm{C}$ is only a dependence on the choice of the reference temperature, and the appearance of shallow minima of $p_{T}$, mostly found for G201 specimens at relatively lower temperatures, corresponds to the inversion point of the temperature-dependence of $K^{\prime}$ and $-K^{\prime \prime}$ maxima mentioned above.

Significancc of the Vertical Shift Factor $p_{T}$. The temperature-dependence of the vertical shift factor $p_{T}$ has been only rarely studied in a 
T. Kyu, N. Yasuda, S. Suehiro, S. Nomura, and H. Kawai

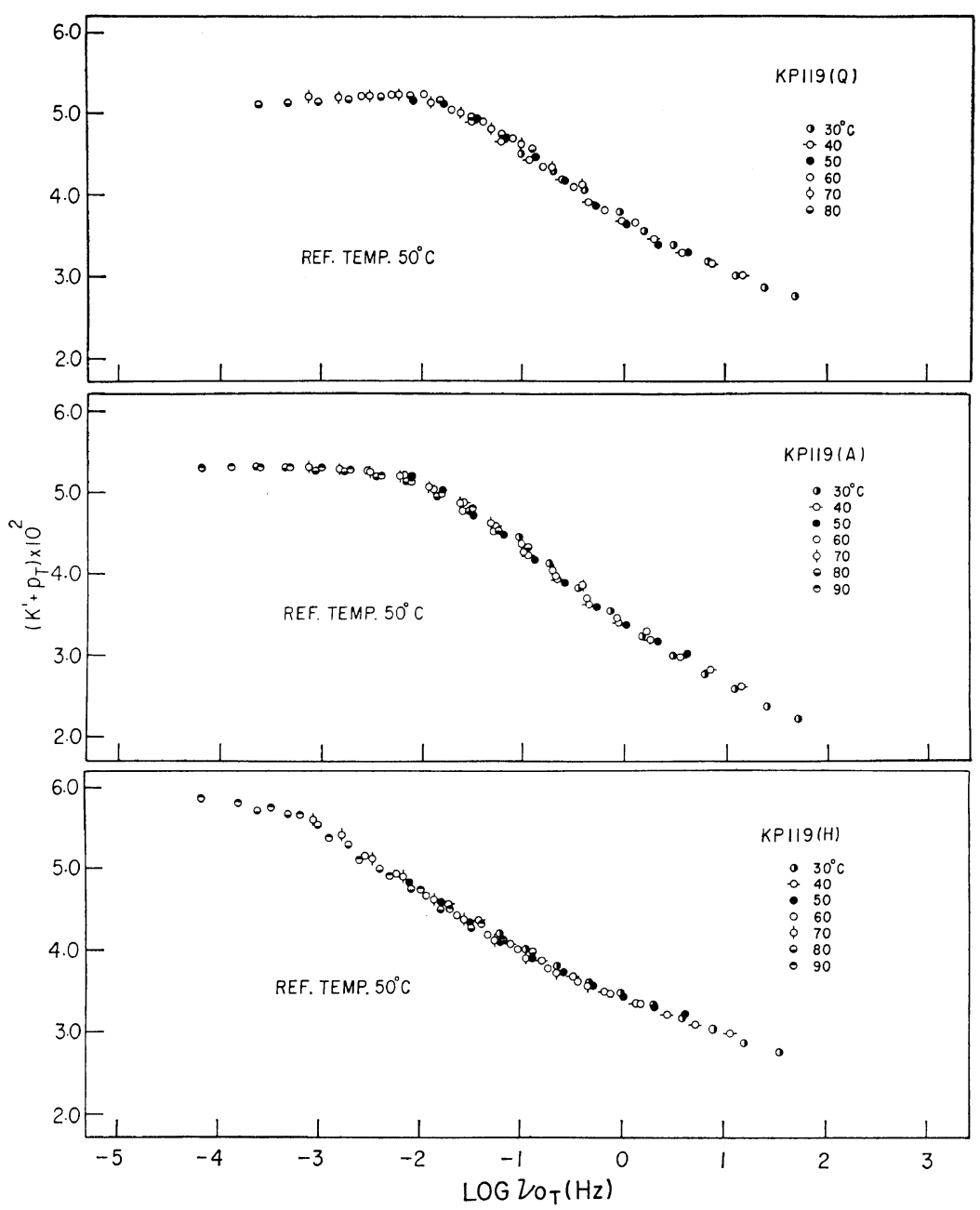

Figure 14. Master curves of the real part of the complex dynamic strain-optical coefficient for a series of medium-density polyethylenes, all reduced to a given reference temperature of $50^{\circ} \mathrm{C}$.

quantitative manner, except for a series of studies by Onogi et al. for a high-density polyethylene $e^{48,49}$ in which the authors have concluded that the temperature-dependence mostly results from that of the orientation compliance $^{26}$ (or the strain-induced orientation coefficient $)^{50}$ of the crystal $c$-axis of the polyethylene crystallites and, further, from that of the mobile fraction of noncrystalline chain segments, but not from that of the degree of crystallinity. The conclusion is actually based on the simplifications that the system be monophase, neglecting the optical contribution from the non- crystalline phase, and that the crystal orientation be represented by that of the crystal $c$-axis. The objection to the temperature dependence of the degree of crystallinity seems not to be the case for the other kinds of polyethylenes, especially for a low-density polyethylene. Hence, let us discuss the problem in more general terms for a particular low-density polyethylene, G201(A), for which the dynamic X-ray diffraction behavior has been fully studied in this laboratory. ${ }^{51}$

Assuming the semicrystalline polymers to be a two-phase system, the measured complex 
Dynamic Birefringence of Semicrystalline Polymers

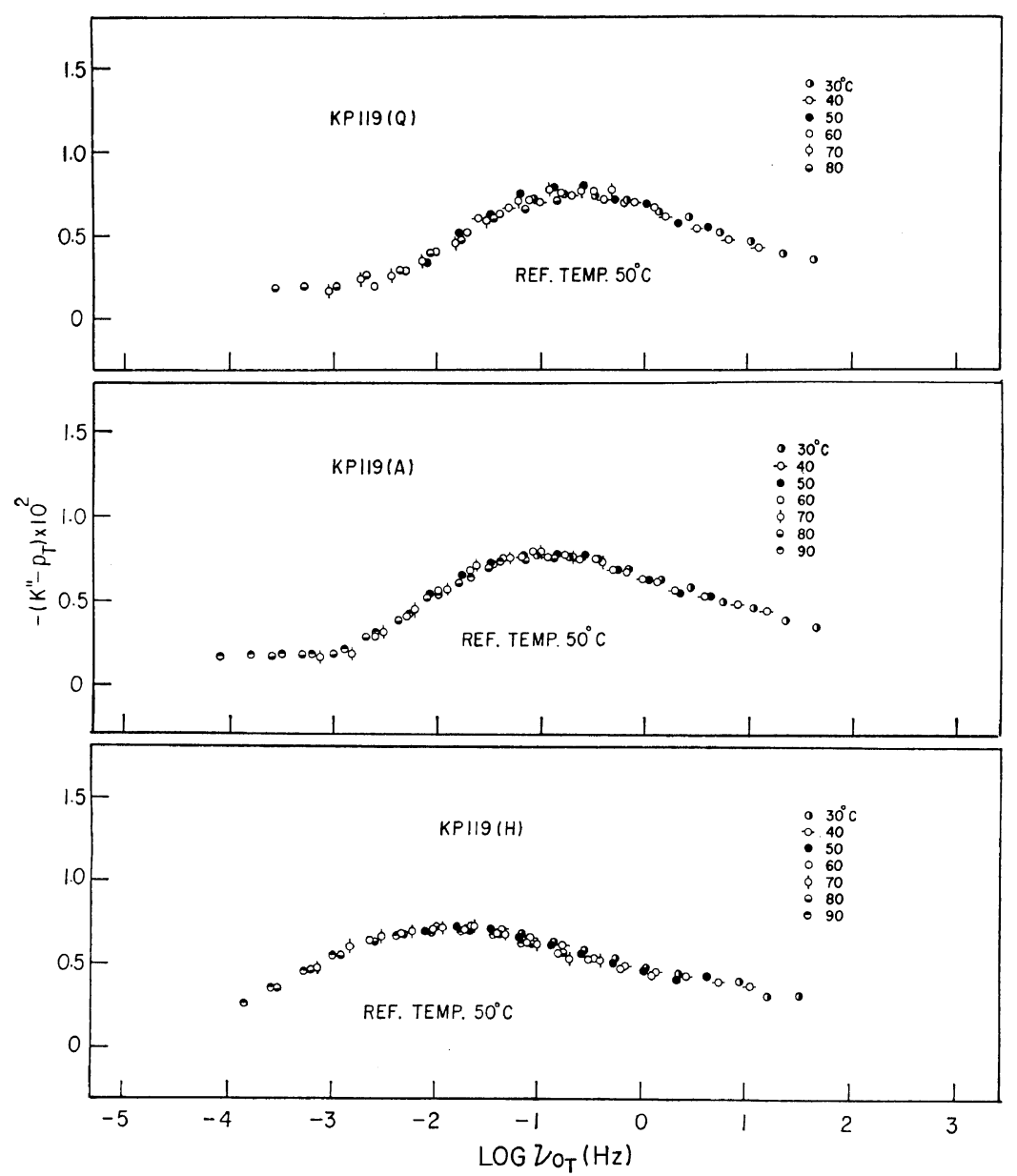

Figure 15. Master curves of the imaginary part of the complex dynamic strain-optical coefficient for a series of medium-density polyethylenes, all reduced to a given reference temperature of $50^{\circ} \mathrm{C}$.

dynamic strain-optical coefficient $K^{*}$ can be written for polyethylene as ${ }^{52}$

$$
\begin{aligned}
K^{*}= & X_{\mathrm{c}}\left[\left(n_{a}-n_{c}\right) C_{a}{ }^{*}+\left(n_{b}-n_{c}\right) C_{b}{ }^{*}\right] \\
& +\left(1-X_{\mathrm{c}}\right) \Delta_{\mathrm{am}}^{0} C_{\mathrm{am}}^{*}+\left(\partial \Delta_{\text {form }} / \partial \varepsilon\right)
\end{aligned}
$$

where $X_{\mathrm{c}}$ is the volume fraction of crystalline phase, $\left(n_{i}-n_{c}\right)$ and $\Delta_{\mathrm{am}}^{0}$ are the intrinsic birefringences of the crystalline and noncrystalline phases, respectively, $C_{i}{ }^{*}$ is the complex dynamic strain-induced orientation coefficients, and the subscripts, $a, b, c$, and am denote the crystal $a, b$, and $c$ axes, and noncrystalline chain segment, respectively. The last term in eq 11 is the contribution from the form birefringence, which is assumed to be negligible. One can expect that every term in eq 11 must be expressed as functions of temperature and frequency, but possibly the crystallinity $X_{\mathrm{c}}$ and the intrinsic birefringences, $\left(n_{i}-n_{c}\right)$ and $\Delta_{\mathrm{am}}^{0}$, are independent of frequency. Thus, eq $11 \mathrm{can}$ be rewritten as a function of temperature $T$ and frequency $\nu$ into

$$
\begin{aligned}
{K_{T}}^{*}(\nu)= & X_{\mathrm{c}}(T) \sum_{i}^{a, b}\left(n_{i}-n_{\mathrm{c}}\right)_{T} C_{i, T}^{*}(\nu) \\
& +\left[1-X_{\mathrm{c}}(T)\right] \Delta_{\mathrm{am}, T}^{0} C_{\mathrm{am}, T}^{*}(\nu)
\end{aligned}
$$

where $C_{i, T}^{*}(\nu)$ can be further written as

$$
C_{i, T}^{*}(\nu)=C_{i, T_{0}}^{*}\left(\nu c_{T_{i}}\right)-d_{T_{i}}^{*}
$$


T. Kyu, N. Yasuda, S. Suehiro, S. Nomura, and H. Kawai

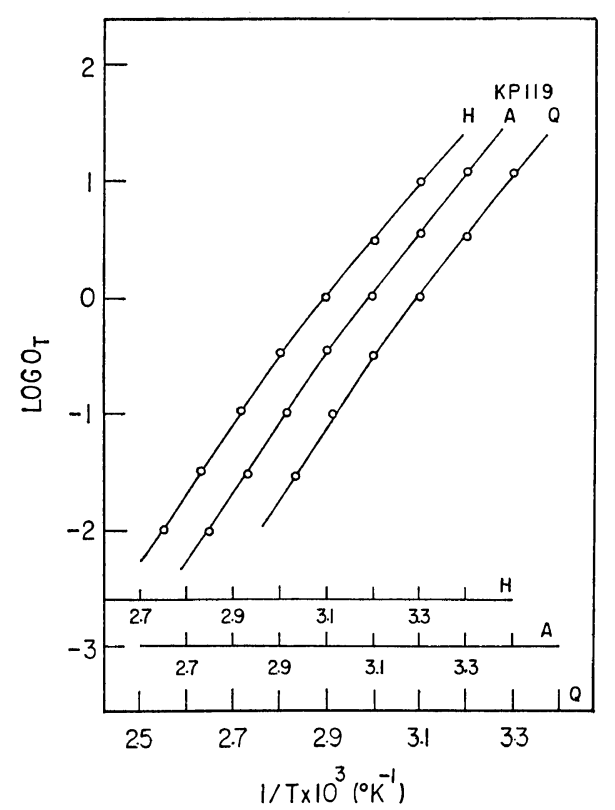

Figure 16. Temperature dependence of the horizontal shift factor $o_{T}\left(T, T_{0}\right)$ for constructing master curves of the complex dynamic strain-optical coefficient for a series of medium-density polyethylenes, taking the reference temperature as $50^{\circ} \mathrm{C}$.

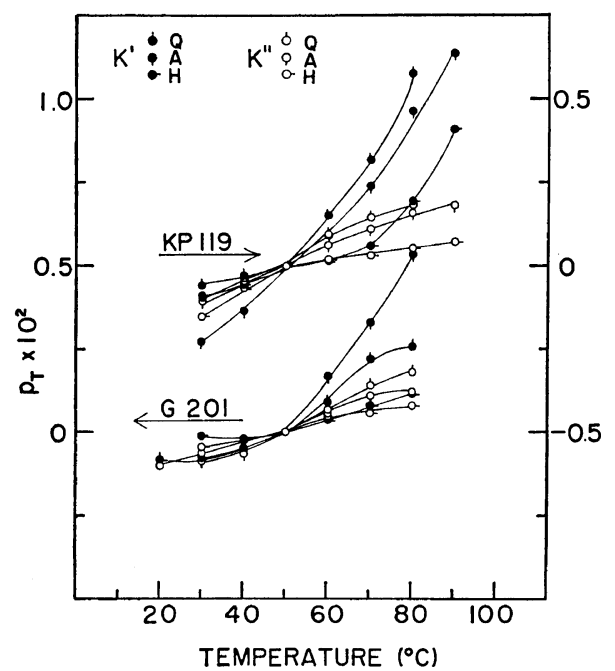

Figure 17. Temperature dependence of the vertical shift factor $p_{T}\left(T, T_{0}\right)$ for constructing master curves of the complex dynamic strain-optical coefficient for two series of low and medium-density polyethylenes, taking the reference temperature as $50^{\circ} \mathrm{C}$.

$$
C_{i, T_{0}}^{*}\left(\nu o_{T}\right)=C_{i, T_{0}}^{*}\left(\nu c_{T_{i}}\right)+\left[C_{i, T_{0}}^{*}\left(\nu o_{T}\right)-C_{i, T_{0}}^{*}\left(\nu c_{T_{i}}\right)\right]
$$

where $c_{T_{i}}$ and $d_{T_{i}}^{*}$ are the horizontal and vertical shift factors for constructing the master curve of the respective dynamic orientation coefficient, $C_{i}{ }^{*}$.

The vertical shift factor $p_{T}$, which is defined by $K_{T_{0}}^{*}\left(\nu O_{T}\right)-K_{T}{ }^{*}(\nu)$, can be given by

$$
\begin{aligned}
p_{T}= & \sum_{i}^{a, b} c_{i, T_{0}}^{*}\left(\nu c_{T_{i}}\right)\left[X_{\mathrm{c}}\left(T_{0}\right)\left(n_{i}-n_{c}\right)_{T_{0}}\right. \\
& \left.-X_{\mathrm{c}}(T)\left(n_{i}-n_{c}\right)_{T}\right]+X_{\mathrm{c}}\left(T_{0}\right) \sum_{i}^{a, b}\left(n_{i}-n_{c}\right)_{T_{0}} \\
& \times\left[C_{i, T_{0}}^{*}\left(\nu o_{T}\right)-C_{i, T_{0}}^{*}\left(\nu c_{T_{i}}\right)\right] \\
& +X_{\mathrm{c}}(T) \sum_{i}^{a, b}\left(n_{i}-n_{c}\right)_{T} d_{T_{i}}^{*} \\
& +C_{\mathrm{am}, T_{0}}^{*}\left(\nu c_{T_{\mathrm{am}}}\right)\left[\left\{1-X_{\mathrm{c}}\left(T_{0}\right)\right\} \Delta_{\mathrm{am}, T_{0}}^{0}\right. \\
& \left.-\left\{1-X_{\mathrm{c}}(T)\right\} \Delta_{\mathrm{am}, T}^{0}\right] \\
& +\left\{1-X_{\mathrm{c}}\left(T_{0}\right)\right\} \Delta_{\mathrm{am}, T_{0}}^{0}\left[C_{\mathrm{am}, T_{0}}^{*}\left(\nu o_{T}\right)\right. \\
& \left.-C_{\mathrm{am}, T_{0}}^{*}\left(\nu c_{T_{\mathrm{am}}}\right)\right]+\left\{1-X_{\mathrm{c}}(T)\right\} \Delta_{\mathrm{am}, T}^{0} d_{T_{\mathrm{am}}}^{*}
\end{aligned}
$$

Equation 14 is a general expression of the vertical shift factor $p_{T}$ on the basis of the bi-shase hypothesis with different $C_{i}{ }^{*}$, and reveals that the vertical shift factor is not only temperaturedependent but also frequency-dependent, which is a self-contradiction to the experimental definition of $p_{T}$ in a strict sense.

Let us further discuss the vertical shift factor under certain conditions. First, let us assume $c_{T_{i}}=o_{T}, d_{T_{i}}^{*}=0$, and that the intrinsic birefringences are temperature-independent; then eq 14 reduces to

$$
\begin{aligned}
p_{T}{ }^{\prime}= & \frac{X_{\mathrm{c}}\left(T_{0}\right)-X_{\mathrm{c}}(T)}{X_{\mathrm{c}}\left(T_{0}\right)}\left[K_{T_{0}}^{\prime}\left(\nu o_{T}\right)\right. \\
& \left.-\Delta_{\mathrm{am}, T_{0}}^{0} C_{\mathrm{am}, T_{0}}^{\prime}\left(\nu o_{T}\right)\right] \\
p_{T}{ }^{\prime \prime}= & \frac{X_{\mathrm{c}}\left(T_{0}\right)-X_{\mathrm{c}}(T)}{X_{\mathrm{c}}\left(T_{0}\right)}\left[K_{T_{0}}^{\prime \prime}\left(\nu o_{T}\right)\right. \\
& \left.-\Delta_{\mathrm{am}, T_{0}}^{0} C_{\mathrm{am} T_{0}}^{\prime \prime}\left(\nu o_{T}\right)\right]
\end{aligned}
$$

Second, let us assume $X_{\mathrm{c}}\left(T_{0}\right)=X_{\mathrm{c}}(T), c_{T_{i}}=o_{T}$, $d_{T_{i}}^{*}=0, \Delta_{\mathrm{am}, T_{0}}^{0}=\Delta_{\mathrm{am}, T}^{0}$, and $n_{c, T_{0}}=n_{c, T} ;$ then eq 14 reduces to

$$
\begin{aligned}
p_{T}= & X_{\mathrm{c}}\left(T_{0}\right)\left[\left(n_{a, T_{0}}-n_{a, T}\right) C_{a, T_{0}}^{*}\left(\nu o_{T}\right)\right. \\
& \left.+\left(n_{b, T_{0}}-n_{b, T}\right) C_{b, T_{0}}^{*}\left(\nu o_{T}\right)\right]
\end{aligned}
$$

Third, let us assume $c_{T_{i}}=o_{T}$, and that the intrinsic birefringences are temperature-independent; then eq 14 reduces to

Polymer J., Vol. 8, No. 6, 1976 


$$
\begin{aligned}
p_{T}= & \sum_{i}^{a . b}\left(n_{i}-n_{\mathrm{c}}\right)_{T_{0}}\left[\left\{X_{\mathrm{c}}\left(T_{0}\right)-X_{\mathrm{c}}(T)\right\} C_{i, T_{0}}^{*}\left(\nu O_{T}\right)\right. \\
& \left.+X_{\mathrm{c}}(T) d_{T_{i}}^{*}\right]+\Delta_{\mathrm{am}, T_{0}}^{0}\left[\left\{X_{\mathrm{c}}(T)\right.\right. \\
& \left.\left.-X_{\mathrm{c}}\left(T_{0}\right)\right\} C_{\mathrm{am}, T_{0}}^{*}\left(\nu O_{T}\right)+\left\{1-X_{\mathrm{c}}(T)\right\} d_{T_{\mathrm{am}}}^{*}\right]
\end{aligned}
$$

If one further assumes $X_{\mathrm{c}}\left(T_{0}\right)=X_{\mathrm{c}}(T)$, then eq 17 reduces to

$$
\begin{aligned}
p_{T}= & \sum_{i}^{a . b}\left(n_{i}-n_{c}\right)_{T_{0}} X_{\mathrm{c}}\left(T_{0}\right) d_{T_{i}}^{*} \\
& +\Delta_{\mathrm{am}, T_{0}}^{0}\left\{1-X_{\mathrm{c}}\left(T_{0}\right)\right\} d_{T_{\mathrm{am}}}^{*}
\end{aligned}
$$

Equation 18 may correspond to the conclusion given by Onogi, et al., ${ }^{49}$ for a high-density polyethylene for which the temperature-dependence of the vertical shift factor $p_{T}$ arises mostly from the temperature-dependence of the orientation compliance $C_{i}{ }^{*}$ through $d_{T_{i}}^{*}$, as $E_{j}{ }^{*}$ arises though $f_{j}\left(T, T_{0}\right)$ in eq 1 and 2 ; i.e., by the ease of the crystal orientation associated with the temperature-dependence of the mobile fraction of surrounding medium, though their definition of $p_{T}$ is in the usual way as the ratio of $\left[C_{T}{ }^{*}(\nu) / C_{T_{0}}^{*}\left(\nu o_{T}\right)\right]$ and is thus different from our definition.

Dynamic X-ray diffraction studies of orientation responses of crystallites in particular lowdensity polyethylenes have revealed that the strain-induced orientation coefficient, $C_{i, T}^{*}(\nu)$, can be superposed without any vertical shift and with a common horizontal shift factor $c_{T}$, irrespective of $i .^{26,51}$ That is:

$$
C_{i, T}^{*}(\nu)=C_{i, T_{0}}^{*}\left(\nu c_{T}\right)
$$

where $i=a$ and $b$. Assuming similarly that the strain-induced orientation coefficient, $C_{\mathrm{am}, T}^{*}(\nu)$, can be superposed without any vartical shift, then

$$
C_{\mathrm{am}, T}^{*}(\nu)=C_{\mathrm{am}, T_{0}}^{*}\left(\nu c_{T_{\mathrm{am}}}\right)
$$

Figure 18 shows the temperature-dependences of the horizontal shift factors $c_{T_{i}}$ and of the volume fraction of crystalline phase $X_{\mathrm{c}}(T)$ obtained from dynamic and static X-ray diffraction measurements, respectively, for the G201(A) specimen. $^{51}$ As can be seen in the figure, the temperature-dependence of $c_{T_{i}}$ is represented by a straight line, irrespective of $i$, when its logarithmic value is plotted against the reciprocal to absolute temperature. From the

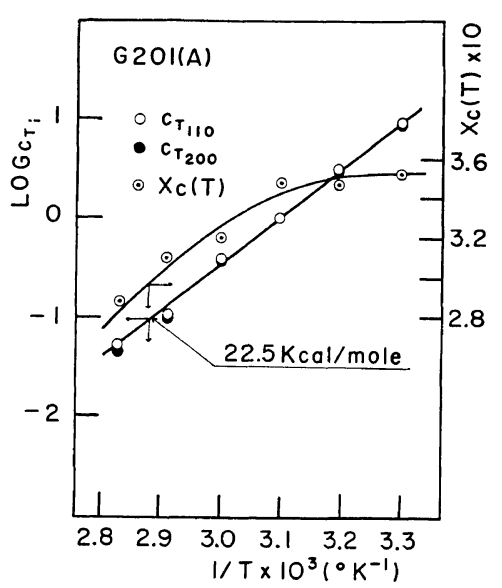

Figure 18. Temperature dependences of the horizontal shift factor $c_{T_{i}}\left(T, T_{0}\right)$ for constructing master curves of the complex dynamic strain-induced orientation coefficient (orientation compliance) of the crystal $i$ th axis and of the fractional volume of the crystalline phase $X_{\mathrm{c}}(T)$ for annealed lowdensity polyethylene.

slope of this straight line, the activation energy of the relaxation process of the crystal orientation is found to be $22.5 \mathrm{kcal} / \mathrm{mol}$, which is very close to those obtained from the temperature-dependences of $o_{T}$ as well as of $a_{T}$, as shown in Figure 8. This fact suggests that $c_{T_{i}} \cong o_{T}$, where $i,=a, b$, and am, and the validities of eq 19 and 20 , if any, also suggest that $d_{T_{i}}^{*} \cong 0$, both showing further the validity of eq 15 for this particular system of G201(A). In other words, the vertical shift of $K_{T}{ }^{*}(\nu)$ for constructing $K_{T_{0}}^{*}\left(\nu O_{T}\right)$, results from the temperature-dependence of the volume fraction of the crystalline phase, $X_{\mathrm{c}}(T)$.

Figure 19 shows the results of the temperature-dependence of the complex dynamic strainoptical coefficient of the noncrystalline phase, $K_{\mathrm{am}, T}^{*}(\nu)$, i.e., $\Delta_{\mathrm{am}, T_{0}}^{0} C_{\mathrm{am}, T}^{\prime}(\nu)$ and $\Delta_{\mathrm{am}, T_{0}}^{0} C_{\mathrm{am}, T}^{\prime \prime}(\nu)$, separated from $K_{T}{ }^{*}(\nu)$ as given in Figure 10 on the basis of eq 15 by using the dynamic X-ray diffraction data, $C_{i, T_{0}}^{*}\left(\nu c_{T_{i}}\right){ }^{51}$ It must be emphasized, first, that $C_{\mathrm{am}, T}^{\prime \prime}(\nu)$ remain almost zero within experimental error. That is, the optical response of the noncrystalline chain segments is well in-phase with the dynamic bulk strain, which is in contradiction to the much delayed response of the crystal orientation, 


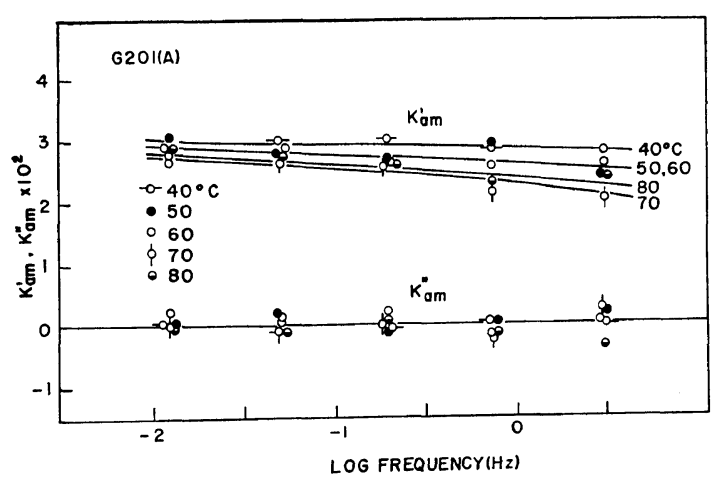

Figure 19. The temperature dependence of the real and imaginary parts of the complex dynamic strain-optical coefficient of noncrystalline phase, $K_{\mathrm{am}, T}^{\prime}(\nu)$ and $K_{\mathrm{am}, T}^{\prime \prime}(\nu)$, for annealed low-density polyethylene, separated from $K_{T}^{*}(\nu)$ in Figure 10.

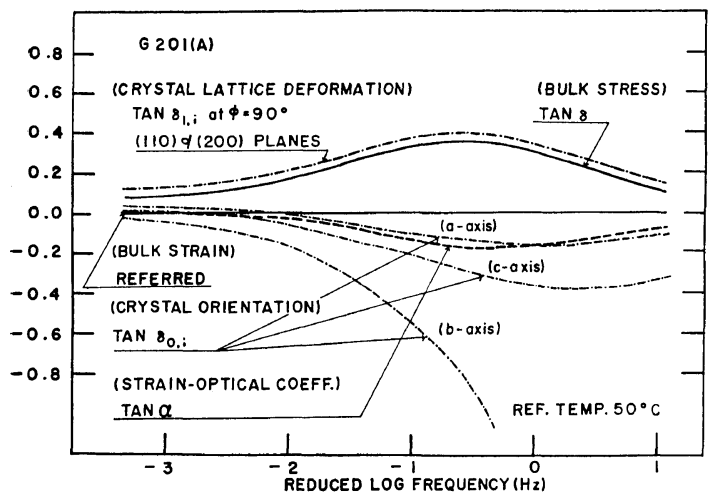

Figure 20. Collected results of the dynamic mechanical and optical responses of annealed lowdensity polyethylene in terms of the frequency dispersions of loss angle tangent functions, all with respect to the bulk strain and reduced to a reference temperature of $50^{\circ} \mathrm{C}$.

as will be shown later in Figure 20. On the other hand, the temperature dependence of $C_{\mathrm{am}, T}^{*}(\nu)$, especially of $C_{\mathrm{am}, T}^{\prime}(\nu)$ thus separated, is quite unexpected in the sense that the data at lower temperatures must be shifted, in general, to the lower frequency side to construct the master curve of $C_{\mathrm{am}, T_{0}}^{\prime}\left(\nu c_{T_{\mathrm{am}}}\right)$; this is opposite to the assumption of $c_{T_{i}} \cong o_{T}$, which requires the shift to be performed to the higher frequency side. This contradiction must be explained in terms

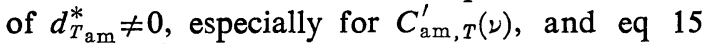
must be rewritten as

$$
\begin{aligned}
p_{T}{ }^{\prime}= & \frac{X_{\mathrm{c}}\left(T_{0}\right)-X_{\mathrm{c}}(T)}{X_{\mathrm{c}}(T)}\left[K_{T_{0}}^{\prime}\left(\nu o_{T}\right)\right. \\
& \left.-\Delta_{\mathrm{am}, T_{0}}^{0} C_{\mathrm{am}, T_{0}}^{\prime}\left(\nu O_{T}\right)\right] \\
& +\left[1-X_{\mathrm{c}}(T)\right] \Delta_{\mathrm{am}, T_{0}}^{0} d_{T_{\mathrm{am}}}^{\prime} \\
p_{T}{ }^{\prime \prime}= & \frac{X_{\mathrm{c}}\left(T_{0}\right)-X_{\mathrm{c}}(T)}{X_{\mathrm{c}}(T)}\left[K_{T_{0}}^{\prime \prime}\left(\nu o_{T}\right)\right. \\
& \left.-\Delta_{\mathrm{am}, T_{0}}^{0} C_{\mathrm{am}, T_{0}}^{\prime \prime}\left(\nu O_{T}\right)\right] \\
& +\left[1-X_{\mathrm{c}}(T)\right] \Delta_{\mathrm{am}, T_{0}}^{0} d_{T_{\mathrm{am}}}^{\prime \prime}
\end{aligned}
$$

where $d_{T_{\mathrm{am}}}^{\prime \prime} \cong 0$.

Figure 20 shows the collected results of the dynamic mechanical and optical responses of the G201(A) specimen in terms of the frequency dispersion of loss angle tangent functions, all referred to the dynamic bulk strain and reduced to the reference temperature of $50^{\circ} \mathrm{C}$. The dynamic crystal lattice response, which is also obtained from the dynamic X-ray diffraction measurements, ${ }^{51}$ is added. It should first be noticed that the mechanical and optical dispersions represented by $\tan \delta$ and $\tan \alpha$, respectively, are definitely associated with the dispersion of crystal orientation given by $\tan \delta_{0, i}$, though the peak frequencies of $\tan \delta_{0, i}$ shift to somewhat higher frequencies than those of $\tan \delta$ and $\tan \alpha$, and that the dynamic lattice deformation of the crystal, represented by $\tan \delta_{1, i}$, is well in-phase with the dynamic bulk stress of the specimen. Actually, the complex dynamic lattice compliance is hardly frequency-dependent, with almost zero imaginary compliance over the ranges of temperature and frequency covered, ${ }^{51}$ and the mechanical dispersion observed here should not be assigned as an $\alpha_{2}$ mechanism.

The most striking problem is, however, how to associate the considerably delayed responses of the crystal orientation, especially that of the crystal $c$-axis, with the noncrystalline orientation which is, by contrast, well in-phase with respect to the bulk strain. The crystal orientation must be primarily governed by that of the noncrystalline chain segments and be, more or less, inphase with that of the chain segments directly connected to the crystals. In other words, there should be at least two distinctive frequency dispersion regions for the noncrystalline orientation responses. One is associated with the delayed crystal responses over the ranges covered, and the other is associated with the primary dispersion, 
i.e., the $\beta$ dispersion appearing at higher frequencies and lower temperatures than the ranges covered here. The former noncrystalline dispersion may be masked in $K_{\mathrm{am}}^{*}$ in Figure 19 by the latter response, except for the appearance of a slight frequency dependence of $K_{\mathrm{am}, T}^{\prime}(\nu)$. Although the interrelation between the former and latter noncrystalline dispersions may differ from specimen to specimen, mostly depending on the microstructure of the noncrystalline region in relation to the crystalline texture, the former dispersion associated with the delayed crystal orientation, if separated, must be understood as the origin of the grain boundary phenomena, i.e., the $\alpha_{1}$ mechanism. A little more quantitative discussion in relation to the mechanical dispersion and the deformation mechanism of spherulitic texture of the material will be presented elsewhere together with original data of the dynamic X-ray diffraction study. ${ }^{51}$

The present definition of $p_{T}$, i.e., $K_{T_{0}}^{*}\left(\nu O_{T}\right)-$ $K_{T}{ }^{*}(\nu)$, seems to be unavoidable for the bulk quantities of $K^{\prime}$ and $-K^{\prime \prime}$ which have no guarantee of keeping their values in the same sign. However, the definition comes out as a selfcontradiction, as discussed just after eq 14 , in a strict sense, simply because of the two-phase hypothesis in which different kinds of optical relaxation mechanisms are assumed with different temperature-dependences of $o_{T_{i}}$ and $d_{T_{i}}$. Therefore, the vertical shift factor must be defined in terms of $p_{T_{j}}=K_{j, T}^{*}(\nu) / K_{j, T_{0}}^{*}\left(\nu O_{T_{j}}\right)$ for each mechanism, as for $f_{j}\left(T, T_{0}\right)$ in eq 1 and 2 , not in terms of $p_{T}=K_{T}{ }^{*}(\nu) / K_{T_{0}}^{*}\left(\nu o_{T}\right)$, for further quantitative discussions. The most significant step is to investigate $p_{T_{j}}$, especially for the noncrystalline orientation compliances $C_{\mathrm{am}, T}^{*}(\nu)$ associated with the $\alpha$ and $\beta$ dispersions separately and to understand the temperature-dependence of $p_{T}$ given by other authors ${ }^{49}$ in terms of a more quantitative approach. Some work along this line is now being carried out by using the series of KP119 for which the contribution from the $\beta$ dispersion seems to be more obvious than the series of G201 specimens.

\section{REFERENCES}

1. H. Nakayasu, H. Markovitz, and D. J. Plazek, Trans. Soc. Rheol., 5, 261 (1961).
2. A. H. Scott, D. J. Scheiber, A. J. Curtis, J. I. Lauritzen, Jr., and J. D. Hoffman, J. Res. Nat. Bur. Stand., A, 66, 269 (1962).

3. S. Iwayanagi and H. Nakane, Rep. Progr. Polym. Phys., Japan, 7, 179 (1964).

4. T. Aramaki, S. Minami, F. Nagatoshi, and M. Takayanagi, ibid., 7, 237 (1964).

5. M. Takayanagi, S. Minami, K. Neki, and A. Nagai, J. Soc. Materials Sci. (Japan), 14, 343 (1965).

6. M. Takayanagi, Proc. 4th Intern. Congr. Rheol., 1, 161 (1965).

7. N. G. McCrum and E. L. Morris, Proc. Roy. Soc. Ser. A (London), 292, 506 (1966).

8. K. M. Sinnott, J. Appl. Phys., 37, 3385 (1966); J. Polym. Sci., Part C, 14, 141 (1966).

9. M. Takayanagi, Pure Appl. Chem., 15, 555 (1967).

10. M. Takayanagi and T. Matsuo, J. Macromol. Sci.-Phys., B1, 407 (1967).

11. N. Saito, K. Okano, S. Iwayanagi, and $T$. Hideshima, in "Solid State Physics", H. Ehrenreich, F. Seitz, and D. Turnbull Eds., Vol. 14, Academic Press, New York, 1963, p 458 .

12. S. Iwayanagi, paper presented at the 2 nd Kyoto Seminor on Polymers, Kyoto, 1968.

13. R. Hayakawa and Y. Wada, Rep. Progr. Polym. Phys., Japan, 9, 193 (1966).

14. K. Okano, Sci. Pap. Inst. Phys. Chem. Res. (Tokyo), 40, 295 (1964); J. Poly. Sci., Part C, 15, 95 (1966).

15. K. Tsuge, H. Enjoji, H. Terada, Y. Ozawa, and Y. Wada, Jap. J. Appl. Phys., 1, 270 (1962).

16. S. Iwayanagi and I. Miura, ibid., 4, 94 (1965).

17. Y. Ishida and K. Yamafuji, Kolloid-Zh., 202, 26 (1965).

18. C. A. F. Tuijnman, Polymer, 4, 259, 315 (1968).

19. P. J. Phillips, G. L. Wilkes, B. W. Delf, and R. S. Stein, J. Polym. Sci., Part A-2, 9, 499 (1971).

20. K. H. Illers, Rheol. Acta, 3, 202 (1964).

21. J. C. Hoffman, C. Williams, and E. Passaglia, J. Polym. Sci., Part C, 14, 173 (1966).

22. T. Hideshima and M. Kakizaki, J. Macromol. Sci.-Phys., B8, 368 (1973).

23. H. Kawai, T. Ito, D. A. Keedy, and R. S. Stein, J. Polym. Sci., Part B, 2, 1075, (1964).

24. T. Ito, T. Oda, H. Kawai, T. Kawaguchi, D. A. Keedy, and R. S. Stein, Rev. Sci. Instr., 39, 1847 (1968).

25. T. Kawaguchi, T. Ito, H. Kawai, D. A. Keedy, and R. S. Stein, Macromolecules, 1, 126 (1968). 
26. A. Tanaka, E. P. Chang, B. Delf, I. Kimura, and R. S. Stein, J. Polym. Sci., Polym. Phys. Ed., 11, 1891 (1973).

27. H. Kawai, T. Ito, and S. Suehiro, paper presented at the 18th Annual Symposium on Rheology, Japan, Odawara, Oct. 8, 1969; Mem. Fac. Eng., Kyoto Univ., 32, 416 (1970).

28. P. Erhardt and R. S. Stein, J. Polym. Sci., Part B, 3, 553 (1965).

29. T. Hashimoto, R. E. Prud'homme, D. A. Keedy, and R.S. Stein, ibid., Polymer Phys. Ed., 11, 693 (1973).

30. P. Erhardt and R. S. Stein, J. Appl. Polym. Sci., Applied Polymer Symposia, 5, 113 (1967).

31. T. Hashimoto, R. E. Prud'homme, and R.S. Stein, J. Polym. Sci., Polymer Phys. Ed., 11, 709 (1973).

32. T. Kyu, N. Yasuda, M. Tabushi, S. Nomura, and H. Kawai, Polymer J., 7, 108 (1975).

33. K. Tajiri, Y. Fujii, M. Aida, and H. Kawai, J. Macromol. Sci.-Phys., B4, 1 (1970).

34. K. Nagamatsu, Kolloid- $Z, 172,141$ (1960).

35. T. Takemura, J. Polym. Sci., 38, 471 (1959).

36. K. Miki, K. Hikichi, and M. Kaneko, Jap. J. Appl. Phys., 6, 931 (1967).

37. K. Miki, J. Yasukawa, and M. Kaneko, ibid., 8, 159 (1969).

38. Y. Wada and K. Tsuge, ibid., 1, 64 (1962).
39. A. Peterlin and E. W. Fischer, Z. Phys., 159, 272 (1960).

40. A. Peterlin, J. Appl. Phys., 31, 1934 (1960).

41. A. Peterlin, E. W. Fischer, and Chr. Reinhold, J. Chem. Phys., 37, 1403 (1962).

42. W. Kuhn and H. Kuhn, Helv. Chim. Acta, 29, 1095 (1946).

43. T. Shimanouchi, M. Asahina, and S. Enomoto, J. Polym. Sci., 59, 93 (1962).

44. B. Szigeti, Proc. Roy. Soc. Ser. A (London), 264, 198 (1961).

45. R. Yamada and R. S. Stein, J. Appl. Phys., 34, 80 (1963).

46. S. Onogi, T. Asada, Y. Fukui, and T. Fujisawa, J. Polym. Sci., Part A-2, 5, 1067 (1967).

47. A. Takeuchi and R. S. Stein, ibid., Part A-2, 5, 1079 (1967).

48. Y. Fukui, T. Sato, M. Ushirokawa, T. Asada, and S. Ongoi, ibid., Part A-2, 8, 1195 (1970).

49. S. Onogi, T. Sato, T. Asada, and Y. Fukui, ibid., Part A-2, 8, 1211 (1970).

50. H. Kawai, Rheol. Acta, 14, 27 (1975).

51. S. Suehiro, T. Yamada, $\mathbf{H}$. Inagaki, and $\mathbf{H}$. Kawai, to be submitted to J. Polym. Sci., Polym. Physics Ed.

52. R. S. Stein, T. Kawaguchi, I. Kimura, and A. Takeuchi, J. Polym. Sci., Part B, 5, 339 (1967). 